\title{
Carathéodory-Julia type conditions and sym- metries of boundary asymptotics for analytic functions on the unit disk
}

\author{
Vladimir Bolotnikov and Alexander Kheifets \\ Mathematics Subject Classification (2000). 41A05, 32A35. \\ Keywords. Carathéodory-Julia condition, boundary asymptotics, $t_{0}$-isometry.
}

\begin{abstract}
It is shown that the following conditions are equivalent for the generalized Schur class functions $w$ at a boundary point $t_{0} \in \mathbb{T}$ : CarathéodoryJulia type condition of order $n$ ([2], [3]); agreeing of asymptotics from inside and outside of the disk $\mathbb{D}$ up to order $2 n+1$ ([11]); $t_{0}$-isometry of the coefficients of the boundary asymptotics; a certain structured matrix $\mathbb{P}$ constructed from these coefficients being Hermitian ([1]). Some interconnections between these properties are established for more general classes of functions.
\end{abstract}

\section{Introduction}

In this paper we discuss some questions arising in the context of the CarathéodoryJulia theorem. For a point $t_{0}$ on the unit circle $\mathbb{T}$, let

$$
\begin{gathered}
\mathcal{U}_{t_{0}, \varepsilon}:=\left\{z \in \mathbb{D}: 0<\left|z-t_{0}\right|<\varepsilon\right\}, \\
\Gamma_{t_{0}, \alpha, \varepsilon}^{\mathrm{int}}:=\left\{z \in \mathcal{U}_{t_{0}, \varepsilon}:\left|\arg \left(z-t_{0}\right)\right|<\alpha\right\} \quad \text { for } \alpha \in\left(0, \frac{\pi}{2}\right), \\
\Gamma_{t_{0}, \alpha, \varepsilon}^{\mathrm{ext}}:=\left\{z \in \mathbb{C}: \frac{1}{\bar{z}} \in \Gamma_{t_{0}, \alpha, \varepsilon}^{\mathrm{int}}\right\}
\end{gathered}
$$

and

$$
\Gamma_{t_{0}, \alpha, \varepsilon}:=\Gamma_{t_{0}, \alpha, \varepsilon}^{\mathrm{int}} \cup \Gamma_{t_{0}, \alpha, \varepsilon}^{\mathrm{ext}}
$$

We consider $\mathcal{U}_{t_{0}, \varepsilon}$ as a punctured neighborhood and $\Gamma_{t_{0}, \alpha, \varepsilon}$ as a punctured nontangential neighborhood of $t_{0}$ that can be split into interior and exterior parts. The parameter $\varepsilon$ will be dropped from notation. We write $z \widehat{\rightarrow} t_{0}$ if a point $z$ approaches a boundary point $t_{0} \in \mathbb{T}$ staying inside $\Gamma_{t_{0}, \alpha}$ for some $\alpha \in\left(0, \frac{\pi}{2}\right)$ and we will write $z \rightarrow t_{0}$ if $z$ approaches $t_{0}$ unrestrictedly in $\mathbb{D}$ (i.e., staying inside $\mathcal{U}_{t_{0}}$ ). Also, "the 
limit exists" will always mean that the limit is finite. For notational convenience, we use the symbol $a^{*}$ for the complex conjugate of $a \in \mathbb{C}$. The symbol $\mathcal{S}$ will stand for the Schur class of analytic functions $w$ mapping the unit disk into its closure. We start with the classical Carathéodory-Julia theorem [7, 9].

Theorem 1.1. Let $w \in \mathcal{S}, t_{0} \in \mathbb{T}$ and let

$$
d_{w}(z):=\frac{1-|w(z)|^{2}}{1-|z|^{2}}
$$

The following are equivalent:

1. $\widetilde{d}:=\liminf _{z \rightarrow t_{0}} d_{w}(z)<\infty . \quad$ 2. $d:=\lim _{z \rightarrow t_{0}} d_{w}(z)<\infty$.

3. The nontangential limits

$$
w_{0}:=\lim _{z \rightarrow t_{0}} w(z) \quad \text { and } \quad w_{1}:=\lim _{z \rightarrow t_{0}} w^{\prime}(z)
$$

exist and satisfy $\left|w_{0}\right|=1$ and $w_{1} t_{0} w_{0}^{*} \in \mathbb{R}$.

Moreover, if the latter hold, then $\widetilde{d}=d=w_{1} t_{0} w_{0}^{*} \geq 0$.

Higher order analogues of the above results have been presented in [3]. To recall them we first introduce some needed notations and definitions.

Given a function $w$ analytic at $z \in \mathbb{D}$ (not necessarily in the Schur class) and given $n \in \mathbb{Z}_{+}$, let us introduce the Hermitian matrix

$$
\mathbf{P}_{n}^{w}(z):=\left[\frac{1}{i ! j !} \frac{\partial^{i+j}}{\partial z^{i} \partial \bar{z}^{j}} \frac{1-|w(z)|^{2}}{1-|z|^{2}}\right]_{i, j=0}^{n}
$$

which will be referred to as to a Schwarz-Pick matrix (a distinguished property of the Schur class is that for $w \in \mathcal{S}$, the matrix $\mathbf{P}_{n}^{w}(z)$ is always positive semidefinite. We extend this notion to boundary points as follows: given $t_{0} \in \mathbb{T}$ and given a function $w$ analytic on a neighborhood $\mathcal{U}_{t_{0}}$ of $t_{0}$, the boundary Schwarz-Pick matrix is defined by

$$
\mathbf{P}_{n}^{w}\left(t_{0}\right):=\lim _{z \leftrightharpoons t_{0}} \mathbf{P}_{n}^{w}(z)
$$

provided the limit in (1.4) exists. It is clear that once the boundary Schwarz-Pick matrix $\mathbf{P}_{n}^{w}\left(t_{0}\right)$ exists, it is Hermitian (and it is positive semidefinite, if $w \in \mathcal{S}$ ). We denote the lower diagonal entry in the Schwarz-Pick matrix $\mathbf{P}_{n}^{w}(z)$ by

$$
d_{w, n}(z):=\frac{1}{(n !)^{2}} \frac{\partial^{2 n}}{\partial z^{n} \partial \bar{z}^{n}} \frac{1-|w(z)|^{2}}{1-|z|^{2}}
$$

and remark that if $n=0$, then both $\mathbf{P}_{n}^{w}(z)$ and $d_{w, n}(z)$ reduce to $d_{w}(z)$ introduced in (1.1).

Throughout the paper, we will use notation $w_{j}(z):=\frac{w^{(j)}(z)}{j !}$ and we reserve the symbol $w_{j}\left(t_{0}\right)$ for the nontangential boundary limit

$$
w_{j}\left(t_{0}\right):=\lim _{z \rightarrow t_{0}} w_{j}(z)=\lim _{z \rightarrow t_{0}} \frac{w^{(j)}(z)}{j !}
$$


provided the latter limit exists. If $w$ is either analytic at $t_{0} \in \mathbb{T}$ or if it possesses the nontangential boundary limits $(1.6)$ for $j=0, \ldots, 2 n+1$, we let

$$
\begin{aligned}
\mathbb{U}_{n}^{w}\left(t_{0}\right) & :=\left[\begin{array}{cccc}
w_{0}\left(t_{0}\right)^{*} & w_{1}\left(t_{0}\right)^{*} & \ldots & w_{n}\left(t_{0}\right)^{*} \\
0 & w_{0}\left(t_{0}\right)^{*} & \ddots & \vdots \\
\vdots & \ddots & \ddots & w_{1}\left(t_{0}\right)^{*} \\
0 & \ldots & 0 & w_{0}\left(t_{0}\right)^{*}
\end{array}\right], \\
\mathbb{H}_{n}^{w}\left(t_{0}\right):= & {\left[\begin{array}{cccc}
w_{1}\left(t_{0}\right) & w_{2}\left(t_{0}\right) & \ldots & w_{n+1}\left(t_{0}\right) \\
w_{2}\left(t_{0}\right) & w_{3}\left(t_{0}\right) & \ldots & w_{n+2}\left(t_{0}\right) \\
\vdots & \vdots & & \vdots \\
w_{n+1}\left(t_{0}\right) & w_{n+2}\left(t_{0}\right) & \ldots & w_{2 n+1}\left(t_{0}\right)
\end{array}\right] }
\end{aligned}
$$

where the first matrix is of the upper triangular Toeplitz and the second of the Hankel structure. We also introduce the structured matrix

$$
\mathbb{P}_{n}^{w}\left(t_{0}\right)=\mathbb{H}_{n}^{w}\left(t_{0}\right) \boldsymbol{\Psi}_{n}\left(t_{0}\right) \mathbb{U}_{n}^{w}\left(t_{0}\right)
$$

where $\boldsymbol{\Psi}_{n}\left(t_{0}\right)=\left[\Psi_{j \ell}\right]_{j, \ell=0}^{n}$ is the upper triangular matrix

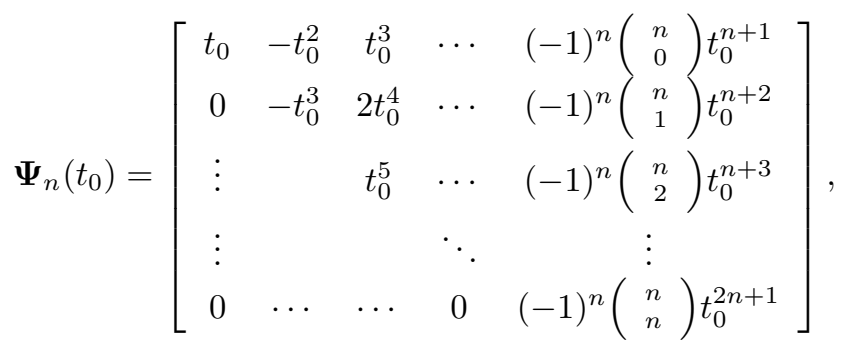

with the entries

$$
\Psi_{j \ell}=\left\{\begin{array}{cl}
0, & \text { if } j>\ell \\
(-1)^{\ell}\left(\begin{array}{c}
\ell \\
j
\end{array}\right) t_{0}^{\ell+j+1}, & \text { if } j \leq \ell .
\end{array}\right.
$$

Also we will make use of similar structured matrices associated with $t_{0} \in \mathbb{T}$ and a sequence $\left\{w_{j}\right\}$ of complex numbers:

$$
\mathbb{U}\left(w_{0}, \ldots, w_{n}\right)=\left[\begin{array}{ccc}
w_{0}^{*} & \ldots & w_{n}^{*} \\
\vdots & \ddots & \vdots \\
0 & \ldots & w_{0}^{*}
\end{array}\right], \quad \mathbb{H}\left(w_{1}, \ldots, w_{2 n+1}\right)=\left[w_{i+j+1}\right]_{i, j=0}^{n}
$$

and

$$
\mathbb{P}\left(t_{0}, w_{0}, \ldots, w_{2 n+1}\right):=\mathbb{H}\left(w_{1}, \ldots, w_{2 n+1}\right) \Psi_{n}\left(t_{0}\right) \mathbb{U}\left(w_{0}, \ldots, w_{n}\right) .
$$

Definition 1.2. Given a point $t_{0} \in \mathbb{T}$, we will say that a sequence $\left\{w_{0}, \ldots, w_{n}\right\}$ of complex numbers is $t_{0}$-isometric if

$$
\overline{\mathbb{U}}\left(w_{0}, \ldots, w_{n}\right) \mathbf{\Psi}_{n}\left(t_{0}\right) \mathbb{U}\left(w_{0}, \ldots, w_{n}\right)=\mathbf{\Psi}_{n}\left(t_{0}\right)
$$


where $\overline{\mathbb{U}}$ is the complex conjugate of $\mathbb{U}$. We will say that an infinite sequence $\left\{w_{i}\right\}_{i=0}^{\infty}$ is $t_{0}$-isometric if the equality (1.14) holds for every $n \in \mathbb{Z}_{+}$.

Remark 1.3. It follows from Definition 1.2 by the upper triangular structure of matrices in (1.14) that if $\left\{w_{0}, \ldots, w_{n}\right\}$ is $t_{0}$-isometric, then the subsequence $\left\{w_{0}, \ldots, w_{k}\right\}$ is $t_{0}$-isometric for every $k<n$. In particular, for $k=0$ we conclude from (1.14) that $\left|w_{0}\right|=1$.

The following theorem has been established in [3].

Theorem 1.4. Let $w \in \mathcal{S}, t_{0} \in \mathbb{T}, n \in \mathbb{Z}_{+}$and let $d_{w, n}$ be defined as in (1.5). The following are equivalent.

1. The following limit inferior is finite

$$
\widetilde{d}_{n}:=\liminf _{z \rightarrow t_{0}} d_{w, n}(z)<\infty .
$$

2. The following nontangential boundary limit exists and is finite:

$$
d_{w, n}\left(t_{0}\right):=\lim _{z \rightarrow t_{0}} d_{w, n}(z)<\infty .
$$

3. The boundary Schwarz-Pick matrix $\mathbf{P}_{n}^{w}\left(t_{0}\right)$ defined via the nontangential boundary limit (1.4) exists.

4. The nontangential boundary limits $w_{j}\left(t_{0}\right)$ exist for $j=0, \ldots, 2 n+1$ and satisfy

$$
\left|w_{0}\left(t_{0}\right)\right|=1 \quad \text { and } \quad \mathbb{P}_{n}^{w}\left(t_{0}\right) \geq 0,
$$

where $\mathbb{P}_{n}^{w}\left(t_{0}\right)$ is the matrix defined in (1.9).

Moreover, if this is the case, then $\widetilde{d}_{n}=d_{w, n}\left(t_{0}\right)$ and $\mathbf{P}_{n}^{w}\left(t_{0}\right)=\mathbb{P}_{n}^{w}\left(t_{0}\right)$.

In case $n=0$, Theorem 1.4 reduces to Theorem 1.1. Note that the structured matrix $\mathbb{P}_{n}^{w}\left(t_{0}\right)$ (which first appeared in [11]) is a higher order analogue of the product $w_{1} t_{0} w_{0}^{*}$. It turns out (and will be shown in this paper) that property (3) in Theorem 1.4 follows from a weaker assumption

$$
\left|w_{0}\left(t_{0}\right)\right|=1 \quad \text { and } \quad \mathbb{P}_{n}^{w}\left(t_{0}\right)=\mathbb{P}_{n}^{w}\left(t_{0}\right)^{*}
$$

even if $w$ does not belong to the Schur class and is analytic on a neighborhood $\mathcal{U}_{t_{0}, \varepsilon}$ only. The following theorem establishes this fact and presents a number of equivalent reformulations of conditions (1.17).

Theorem 1.5. Let $w$ be analytic in a neighborhood $\mathcal{U}_{t_{0}}$ of $t_{0} \in \mathbb{T}$ and let us assume that the nontangential boundary limits $w_{j}\left(t_{0}\right)$ exist for $j=0, \ldots, 2 n+1$. The following are equivalent:

1. Conditions (1.17) are satisfied.

2. The sequence $\left\{w_{0}\left(t_{0}\right), \ldots, w_{2 n+1}\left(t_{0}\right)\right\}$ is $t_{0}$-isometric, i.e.,

$$
\overline{\mathbb{U}}\left(w_{0}, \ldots, w_{2 n+1}\right) \boldsymbol{\Psi}_{n}\left(t_{0}\right) \mathbb{U}\left(w_{0}, \ldots, w_{2 n+1}\right)=\boldsymbol{\Psi}_{2 n+1}\left(t_{0}\right) .
$$

3. There exists a rational unimodular on $\mathbb{T}$ function $f$ (a ratio of two finite Blaschke products) so that

$$
w(z)=f(z)+o\left(\left(z-t_{0}\right)^{2 n+1}\right) \quad \text { as } z \widehat{\rightarrow} t_{0} .
$$


4. The asymptotic relation

$$
w(z)=\sum_{j=0}^{2 n+1} w_{j}\left(t_{0}\right)\left(z-t_{0}\right)^{j}+o\left(\left(z-t_{0}\right)^{2 n+1}\right)
$$

holds as $z$ tends to $t_{0}$ nontangentially from inside and outside of the unit disk $\mathbb{D}$, where for $|z|>1$

$$
w(z):=\frac{1}{\overline{w(1 / \bar{z})}} .
$$

Moreover, if this is the case, then the boundary Schwarz-Pick matrix $\mathbf{P}_{n}^{w}\left(t_{0}\right)$ exists and is equal to $\mathbb{P}_{n}^{w}\left(t_{0}\right)$.

Remark 1.6. Extension (1.20) is called the symmetry continuation and has nothing to do with analytic continuation unless the function $w$ is unimodular on an arc of $\mathbb{T}$. In general, existence of a nontangential asymptotics (1.19) for $w(z)$ from inside of $\mathbb{D}$ with $w\left(t_{0}\right) \neq 0$ implies existence of an asymptotics of the same order from outside for the symmetry continuation of $w$. However, in general, the coefficients of the asymptotics from outside may differ from the coefficients of the asymptotics from inside. Thus, agreeing of the two asymptotics is a special property of the function $w$. Theorem 1.4 tells that this property implies (1.15) and Theorem 1.5 shows that Schur class functions it follows form (1.15). In general (1.15) does not imply this property (see example in Section 4). However, if $w$ is in generalized Schur class then it does. We show in Section 4 that Theorem 1.4 can be literally extended to the class of genearlized Schur functions. The agreeing of the two asymptotics was actually taken by I.V. Kovalishina as a basis for her constructions in [11], [11] as opposed to [3] and [6], where all the constructions started from (1.15).

The next theorem is a version of Theorem 1.5 under slightly relaxed assumptions: existence of the nontangential boundary limit $w_{2 n+1}\left(t_{0}\right)$ is relaxed to the uniform nontangential boundedness of $w^{(2 n+1)}$ :

$$
\sup _{z \in \Gamma_{t_{0}, \alpha}}\left|w^{(2 n+1)}(z)\right|<\infty \quad \text { for some } \alpha \in\left(0, \frac{\pi}{2}\right) .
$$

It can be shown (see Lemma 3.1 below) that the latter condition guarantees the existence of the nontangential boundary limits $w_{j}\left(t_{0}\right):=\lim _{z \rightarrow t_{0}} \frac{w^{(j)}(z)}{j !}$ for $j=$ $0, \ldots, 2 n$.

Theorem 1.7. Let $w$ be analytic in a neighborhood $\mathcal{U}_{t_{0}}$ of $t_{0} \in \mathbb{T}$. The following are equivalent

1. The bound (1.21) is in force and the sequence $\left\{w_{0}\left(t_{0}\right), \ldots, w_{2 n}\left(t_{0}\right)\right\}$ is $t_{0}$ isometric.

2. There exist a rational unimodular function $f$ (a ratio of two finite Blaschke products) so that

$$
w(z)=f(z)+O\left(\left(z-t_{0}\right)^{2 n+1}\right) \quad \text { as } z \widehat{\rightarrow} t_{0} .
$$


3. The asymptotic relation

$$
w(z)=\sum_{j=0}^{2 n} w_{j}\left(t_{0}\right)\left(z-t_{0}\right)^{j}+O\left(\left(z-t_{0}\right)^{2 n+1}\right),
$$

holds as $z \widehat{\rightarrow} t_{0}$ from inside and outside of $\mathbb{D}$.

Moreover, if this is the case, then

$$
\sup _{z \in \Gamma_{t_{0}, \alpha}} d_{w, n}(z)<\infty
$$

As a corollary we obtain the following extended version of Theorem 1.4.

Theorem 1.8. Let $w \in \mathcal{S}, t_{0} \in \mathbb{T}, n \in \mathbb{Z}_{+}$. The statements $1-4$ in Theorem 1.4 , the statements 1-4 in Theorem 1.5 and the statements $1-3$ in Theorem 1.7 are all equivalent.

For the proof it is enough to note that every statement in Theorem 1.5 implies (1.16) while condition (1.23) is obviously stronger than (1.15).

\section{2. $t_{0}$-isometric sequences}

A $t_{0}$-isometric sequence $\left\{w_{0}, \ldots, w_{n}\right\}$ has been characterized in Definition 1.2 by the matrix equality (1.14); in more detail,

$$
\left[\begin{array}{ccc}
w_{0} & \ldots & w_{n} \\
& \ddots & \vdots \\
0 & & w_{0}
\end{array}\right] \boldsymbol{\Psi}_{n}\left(t_{0}\right)\left[\begin{array}{ccc}
w_{0}^{*} & \ldots & w_{n}^{*} \\
& \ddots & \vdots \\
0 & & w_{0}^{*}
\end{array}\right]=\boldsymbol{\Psi}_{n}\left(t_{0}\right) .
$$

Remark 2.1. Note that the latter matrix equality is equivalent to the following $n+1$ (in general, independent) relations

$$
\sum_{j=0}^{k} \sum_{\ell=0}^{k-j}(-1)^{\ell}\left(\begin{array}{c}
k-\ell \\
j
\end{array}\right) t_{0}^{j-\ell} w_{j} w_{\ell}^{*}=1 \quad \text { for } k=0, \ldots, n .
$$

Indeed, multiplying both parts in $(2.2)$ by $(-1)^{k} t_{0}^{k+1}$ and making use of numbers (1.11) we get

$$
\sum_{j=0}^{k} \sum_{\ell=0}^{k-j} w_{j} \Psi_{j, k-\ell} w_{\ell}^{*}=\Psi_{0, k} \text { for } k=0, \ldots, n,
$$

that can be written in the matrix form as

$$
\left[\begin{array}{lll}
w_{0} & \ldots & w_{n}
\end{array}\right] \boldsymbol{\Psi}_{n}\left(t_{0}\right)\left[\begin{array}{ccc}
w_{0}^{*} & \ldots & w_{n}^{*} \\
& \ddots & \vdots \\
0 & & w_{0}^{*}
\end{array}\right]=\left[\begin{array}{lll}
\Psi_{00} & \ldots & \Psi_{0 n}
\end{array}\right]
$$


and express equality of the top rows in (2.1). It was shown in [2] that (2.3) is equivalent to the "whole" equality (2.1).

Lemma 2.2. Let $t_{0} \in \mathbb{T}, w_{0}, \ldots, w_{n} \in \mathbb{C}$ and let $T \in \mathbb{C}^{(n+1) \times(n+1)}$ and $M, E \in$ $\mathbb{C}^{(n+1) \times 1}$ be given by

$$
T_{n}=\left[\begin{array}{cccc}
t_{0} & 0 & \ldots & 0 \\
1 & t_{0} & \ddots & \vdots \\
& \ddots & \ddots & 0 \\
0 & & 1 & t_{0}
\end{array}\right], \quad E_{n}=\left[\begin{array}{c}
1 \\
0 \\
\vdots \\
0
\end{array}\right], \quad M_{n}=\left[\begin{array}{c}
w_{0} \\
w_{1} \\
\vdots \\
w_{n}
\end{array}\right]
$$

The Stein equation

$$
X-T_{n} X T_{n}^{*}=E_{n} E_{n}^{*}-M_{n} M_{n}^{*}
$$

has a solution $X$ if and only if the sequence $\left\{w_{0}, \ldots, w_{n}\right\}$ is $t_{0}$-isometric. Moreover, in this case every solution $X$ to (2.5) is of the form (1.13) for some choice of $w_{n+1}, \ldots, w_{2 n+1} \in \mathbb{C}$.

The proof can be found in [2, Section 10] along with some other equivalent reformulations of the equality (2.1).

Theorem 2.3. Let $w$ be analytic on a neighborhood

$$
D_{r, \varepsilon}\left(t_{0}\right)=\left\{z: r<|z|<\frac{1}{r}, \arg t_{0}-\varepsilon<\arg z<\arg t_{0}+\varepsilon\right\}
$$

of $t_{0} \in \mathbb{T}$ and unimodular on $D_{r, \varepsilon}\left(t_{0}\right) \cap \mathbb{T}$ and let

$$
w(z)=\sum_{j=0}^{\infty} w_{j}\left(t_{0}\right)\left(z-t_{0}\right)^{j}
$$

be its Taylor expansion at $t_{0}$. Then

1. The boundary Schwartz-Pick matrix $\mathbf{P}_{n}^{w}\left(t_{0}\right)$ exists and admits the representation

$$
\mathbf{P}_{n}^{w}\left(t_{0}\right)=\mathbb{P}_{n}^{w}\left(t_{0}\right)
$$

for every $n \in \mathbb{Z}_{+}$, where $\mathbb{P}_{n}^{w}\left(t_{0}\right)$ is defined via formula (1.9). Therefore, $\mathbb{P}_{n}^{w}\left(t_{0}\right)$ is Hermitian.

2. The sequence $\left\{w_{j}\left(t_{0}\right)\right\}_{j=0}^{\infty}$ is $t_{0}$-isometric.

Proof. Since $w$ is unimodular on $D_{r, \varepsilon}\left(t_{0}\right) \cap \mathbb{T}$, its symmetry continuation and analytic continuation across the arc agree and

$$
w(z) \overline{w(1 / \bar{z})}=1 \quad \text { for every } z \in D_{r, \varepsilon}\left(t_{0}\right) .
$$

Therefore,

$$
K_{w}(z, \zeta):=\frac{1-w(z) \overline{w(\zeta)}}{1-z \bar{\zeta}}=\frac{w(z)-w(1 / \bar{\zeta})}{z-\frac{1}{\zeta}} \cdot \frac{\overline{w(\zeta)}}{\bar{\zeta}}
$$


The latter formula makes sense for every $z, \zeta \in D_{r, \varepsilon}\left(t_{0}\right)$ such that $z \bar{\zeta} \neq 1$. We extend it by continuity to all of $D_{r, \varepsilon}\left(t_{0}\right) \times D_{r, \varepsilon}\left(t_{0}\right)$ upon setting

$$
K_{w}(z, 1 / \bar{z})=z w^{\prime}(z) \overline{w(1 / \bar{z})} .
$$

The kernel $K_{w}$ defined by the formulas (2.9) and (2.10) is analytic in $z$ and conjugate analytic in $\zeta$ on $D_{r, \varepsilon}\left(t_{0}\right) \times D_{r, \varepsilon}\left(t_{0}\right)$. Therefore, the boundary Schwarz-Pick matrix $\mathbf{P}_{n}^{w}\left(t_{0}\right)$ can be defined by the formula

$$
\mathbf{P}_{n}^{w}\left(t_{0}\right)=\left[\left.\frac{1}{i ! j !} \frac{\partial^{i+j}}{\partial z^{i} \partial \bar{z}^{j}} K_{w}(z, z)\right|_{z=t_{0}}\right]_{i, j=0}^{n},
$$

rather than as the limit of the interior Schwarz-Pick matrices. To express the $i j$-th entry $\mathbf{P}_{i j}$ in (2.11), we use (2.9) along with the Leibnitz's rule:

$$
\begin{aligned}
\mathbf{P}_{i j} & =\left.\frac{1}{i ! j !} \frac{\partial^{i+j}}{\partial z^{i} \partial \bar{\zeta}^{j}}\left(\frac{w(z)-w(1 / \bar{\zeta})}{z-\frac{1}{\zeta}} \cdot \frac{\overline{w(\zeta)}}{\bar{\zeta}}\right)\right|_{z=\zeta=t_{0}} \\
& =\left.\frac{1}{i ! j !} \sum_{\ell=0}^{j}\left(\begin{array}{c}
j \\
\ell
\end{array}\right) \frac{\partial^{i+\ell}}{\partial z^{i} \partial \bar{\zeta}^{\ell}}\left(\frac{w(z)-w(1 / \bar{\zeta})}{z-\frac{1}{\zeta}}\right) \cdot \overline{\left(\frac{w(\zeta)}{\zeta}\right)^{(j-\ell)}}\right|_{z=\zeta=t_{0}} .
\end{aligned}
$$

We want to show that $\mathbf{P}_{i j}$ is equal to the $i j$-th entry in the matrix $\mathbb{P}_{n}^{w}\left(t_{0}\right)$, i.e. (see the formula (1.9)), that

$$
\begin{aligned}
\mathbf{P}_{i j} & =\left[\begin{array}{llll}
w_{i+1}\left(t_{0}\right) & w_{i+2}\left(t_{0}\right) & \ldots & w_{i+j+1}\left(t_{0}\right)
\end{array}\right] \boldsymbol{\Psi}_{n}\left(t_{0}\right)\left[\begin{array}{c}
w_{j}\left(t_{0}\right)^{*} \\
\vdots \\
w_{0}\left(t_{0}\right)^{*} \\
0
\end{array}\right] \\
& =\sum_{\ell=0}^{j} w_{i+\ell+1}\left(t_{0}\right) \sum_{k=0}^{j-\ell} \Psi_{\ell, j-k} w_{j-\ell-k}\left(t_{0}\right)^{*} .
\end{aligned}
$$

It is easily seen (we refer to $[4$, Section 6$]$ for details) that

$$
\left.\frac{1}{i ! k !} \frac{\partial^{i+k}}{\partial z^{i} \partial\left(\frac{1}{\zeta}\right)^{k}}\left(\frac{w(z)-w(1 / \bar{\zeta})}{z-\frac{1}{\zeta}}\right)\right|_{z=\frac{1}{\zeta}}=\frac{w^{(i+k+1)}(z)}{(i+k+1) !}=w_{i+k+1}(z)
$$

for $i, k \in \mathbb{Z}_{+}$. On the other hand, we have by the chain rule,

$$
\frac{1}{\ell !} \frac{\partial^{\ell}}{\partial \bar{\zeta}^{\ell}}=(-1)^{\ell} \sum_{k=0}^{\ell-1} \frac{\left(\begin{array}{c}
\ell-1 \\
k
\end{array}\right)}{\bar{\zeta}^{\ell+k+1}(k+1) !} \cdot \frac{\partial^{k+1}}{\partial\left(\frac{1}{\zeta}\right)^{k+1}} \quad(\ell=1,2, \ldots)
$$


and combining the two latter formulas gives

$$
\begin{aligned}
& \left.\frac{1}{i ! \ell !} \frac{\partial^{i+\ell}}{\partial z^{i} \partial \bar{\zeta}^{\ell}}\left(\frac{w(z)-w(1 / \bar{\zeta})}{z-\frac{1}{\zeta}}\right)\right|_{z=\zeta=t_{0}} \\
& =\left.(-1)^{\ell} \sum_{k=0}^{\ell-1} \frac{\left(\begin{array}{c}
\ell-1 \\
k
\end{array}\right)}{\bar{\zeta}^{\ell+k+1} i !(k+1) !} \cdot \frac{\partial^{i+k+1}}{\partial z^{i} \partial\left(\frac{1}{\zeta}\right)^{k+1}}\left(\frac{w(z)-w(1 / \bar{\zeta})}{z-\frac{1}{\zeta}}\right)\right|_{z=\zeta=t_{0}} \\
& =(-1)^{\ell} \sum_{k=0}^{\ell-1} t_{0}^{\ell+k+1}\left(\begin{array}{c}
\ell-1 \\
k
\end{array}\right) w_{i+k+2}\left(t_{0}\right)
\end{aligned}
$$

for $\ell=1,2, \ldots$, while for $\ell=0$, we have

$$
\left.\frac{1}{i !} \cdot \frac{\partial^{i}}{\partial z^{i}}\left(\frac{w(z)-w(1 / \bar{\zeta})}{z-\frac{1}{\zeta}}\right)\right|_{z=\zeta=t_{0}}=w_{i+1}\left(t_{0}\right) .
$$

Finally, by the Leibnitz's rule,

$$
\left.\frac{1}{(j-\ell) !}\left(\frac{w(\zeta)}{\zeta}\right)^{(j-\ell)}\right|_{\zeta=t_{0}}=\sum_{m=0}^{j-\ell}(-1)^{m} \bar{t}_{0}^{m+1} w_{j-\ell-m}\left(t_{0}\right)
$$

and we substitute the three latter formulas into (2.12) to get

$$
\begin{aligned}
\mathbf{P}_{i j}= & w_{i+1}\left(t_{0}\right) \sum_{m=0}^{j}(-1)^{m} \bar{t}_{0}^{m+1} w_{j-m}\left(t_{0}\right)^{*} \\
& +\sum_{\ell=1}^{j} \sum_{k=0}^{\ell-1} \sum_{m=0}^{j-\ell}(-1)^{\ell+m} t_{0}^{\ell+k+m+2}\left(\begin{array}{c}
\ell-1 \\
k
\end{array}\right) w_{i+k+2}\left(t_{0}\right) w_{j-\ell-m}\left(t_{0}\right)^{*} \\
= & w_{i+1}\left(t_{0}\right) \sum_{m=0}^{j}(-1)^{m} \bar{t}_{0}^{m+1} w_{j-m}\left(t_{0}\right)^{*} \\
& +\sum_{\ell=1}^{j} w_{i+\ell+1}\left(t_{0}\right) \sum_{k=0}^{j-\ell}(-1)^{j-k} t_{0}^{j+\ell+1-k}\left(\begin{array}{c}
j-k \\
\ell
\end{array}\right) w_{j-\ell-k}\left(t_{0}\right)^{*} \\
= & \sum_{\ell=0}^{j} w_{i+\ell+1}\left(t_{0}\right) \sum_{k=0}^{j-\ell}(-1)^{j-k} t_{0}^{j+\ell+1-k}\left(\begin{array}{c}
j-k \\
\ell
\end{array}\right) w_{j-\ell-k}\left(t_{0}\right)^{*} .
\end{aligned}
$$

Making use of the numbers (1.11) allows us to rewrite the last equality in the form

$$
\mathbf{P}_{i j}=\sum_{\ell=0}^{j} w_{i+\ell+1}\left(t_{0}\right) \sum_{k=0}^{j-\ell} \Psi_{\ell, j-k} w_{j-\ell-k}\left(t_{0}\right)^{*}
$$

which coincides with (2.13) and therefore, proves equality (2.7). This equality implies in particular, that the structured matrix $\mathbb{P}_{n}^{w}\left(t_{0}\right)$ of the form (1.9) is Hermitian 
(since $\mathbf{P}_{n}^{w}\left(t_{0}\right)$ is). Then it follows by [6, Theorem 7.1], that $\mathbb{P}_{n}^{w}\left(t_{0}\right)$ satisfies the Stein identity

$$
\mathbb{P}_{n}^{w}\left(t_{0}\right)-T_{n} \mathbb{P}_{n}^{w}\left(t_{0}\right) T_{n}^{*}=E_{n} E_{n}^{*}-M^{w}\left(t_{0}\right)_{n} M_{n}^{w}\left(t_{0}\right)^{*}
$$

where $T_{n}$ and $E_{n}$ are the same as in (2.4) and where

$$
M_{n}^{w}\left(t_{0}\right)=\left[\begin{array}{lll}
w_{0}\left(t_{0}\right) & \ldots & w_{n}\left(t_{0}\right)
\end{array}\right]^{\top} .
$$

Then, the sequence $\left\{w_{0}, \ldots, w_{n}\right\}$ is $t_{0}$-isometric by Lemma 2.2 . Since $n \in \mathbb{Z}_{+}$is arbitrary, the second assertion of the theorem follows. Note that $t_{0}$-symmetry of the sequance $\left\{w_{0}, \ldots, w_{n}\right\}$ can be derived directly upon differentiating identity (2.8).

Lemma 2.4. Let $t_{0}$ and $w_{0}, \ldots, w_{2 n+1} \in \mathbb{C}$ be such that $\left|w_{0}\right|=1$ and the matrix $\mathbb{P}\left(t_{0} ; w_{0}, \ldots, w_{2 n+1}\right)$ defined in $(1.13)$ is Hermitian. Then there exist $f_{0}, \ldots, f_{2 n+1} \in$ $\mathbb{C}$ with $\left|f_{0}\right|=1$ such that the matrices $\mathbb{P}\left(t_{0} ; f_{0}, \ldots, f_{2 n+1}\right)$ and $\mathbb{P}\left(t_{0} ; s_{0}, \ldots, s_{2 n+1}\right)$ are positive definite where $s_{j}$ 's are the numbers given by

$$
s_{j}=\sum_{\ell=0}^{j} f_{j-\ell} w_{\ell} \quad \text { for } j=0, \ldots, 2 n+1 .
$$

Proof. We will use the following notation for the matrices introduced in (1.12) and (1.13):

$$
\mathbb{U}_{k}^{w}:=\mathbb{U}\left(w_{0}, \ldots, w_{k}\right) \quad \text { and } \quad \mathbb{H}_{k}^{w}:=\mathbb{H}\left(w_{0}, \ldots, w_{2 k+1}\right),
$$

and

$$
\mathbb{P}_{k}^{w}:=\mathbb{P}\left(t_{0} ; w_{0}, \ldots, w_{2 k+1}\right)=\mathbb{H}_{k}^{w} \boldsymbol{\Psi}_{k}\left(t_{0}\right) \mathbb{U}_{k}^{w}
$$

and for the similar matrices associated with the sequences $\left\{f_{j}\right\}$ and $\left\{s_{j}\right\}$. Note that if $s_{j}$ 's are defined by the convolution formulas (2.14), then

$$
\mathbb{U}_{k}^{s}=\mathbb{U}_{k}^{w} \mathbb{U}_{k}^{f} \quad \text { and } \quad \mathbb{H}_{k}^{s}=\left(\mathbb{U}_{k}^{f}\right)^{*} \mathbb{H}_{k}^{w}+\mathbb{H}_{n}^{f}\left(t_{0}\right) \overline{\mathbb{U}_{k}^{s}} .
$$

The desired sequence $\left\{f_{0}, \ldots, f_{2 n+1}\right\}$ will be constructed inductively. Note that for every $k<n$, the matrx $\mathbb{P}_{k}^{w}$ is Hermitian as a principal submatrix of $\mathbb{P}_{n}^{w}$ which is Hermitian by the assumption of the lemma. For $k=0$, we have $\mathbb{P}_{0}^{w}:=t_{0} w_{1} w_{0}^{*} \in \mathbb{R}$. Let $f_{0}$ and $f_{1}$ be such that

$$
\left|f_{0}\right|=1 \quad \text { and } \quad \mathbb{P}_{0}^{f}=t_{0} f_{1} f_{0}^{*}>\max \left\{0,-t_{0} w_{1} w_{0}^{*}\right\}
$$

(such a choice is obviously possible). Then for $s_{0}=f_{0} w_{0}$ and $s_{1}=f_{0} w_{1}+f_{1} w_{0}$, we have $\left|s_{0}\right|=1$ and

$$
\mathbb{P}_{0}^{s}=t_{0} s_{1} s_{0}^{*}=t_{0}\left(f_{0} w_{1}+f_{1} w_{0}\right) f_{0}^{*} w_{0}^{*}=t_{0} w_{1} w_{0}^{*}+t_{0} f_{1} f_{0}^{*}>0 .
$$

Let us assume that for $k<n$ we already have $\left\{f_{0}, \ldots, f_{2 k-1}\right\}$ such that the matrices $\mathbb{P}_{k-1}^{f}$ and $\mathbb{P}_{k-1}^{s}$ are positive definite where $s_{j}$ 's are defined by the formula (2.14) for $j=0, \ldots, 2 k-1$. We will show that for an appropriate choice of $f_{2 k}$ and $f_{2 k+1}$, the extended matrices $\mathbb{P}_{k}^{f}$ and $\mathbb{P}_{k}^{s}$ are positive definite which will complete the proof by induction. 
Since $\left|f_{0}\right|=1$ and $\mathbb{P}_{k-1}^{f}>0$, it follows by an interpolation result (see e.g., [11, 1, $3]$ ), there exists a finite Blaschke product $b(z)$ such that

$$
\frac{b^{(j)}\left(t_{0}\right)}{j !}=f_{j} \quad \text { for } j=0, \ldots, 2 k-1 .
$$

Letting

$$
f_{2 k}=\frac{b^{(2 k)}\left(t_{0}\right)}{(2 k) !} \quad \text { and } \quad f_{2 k+1}=\frac{b^{(2 k+1)}\left(t_{0}\right)}{(2 k+1) !}
$$

define $s_{2 k}$ and $s_{2 k+1}$ according to (2.14). The matrix

$$
\mathbb{P}_{k}^{f}:=\mathbb{H}_{k}^{f} \boldsymbol{\Psi}_{k}\left(t_{0}\right) \mathbb{U}_{k}^{f} .
$$

is positive semidefinite, since it is equal (by construction) to the matrix $\mathbb{P}_{k}^{b}\left(t_{0}\right)$ associated to the finite Blaschke product $b$ via formula (1.9) and therefore, it is equal (by Theorem 2.3) to the boundary Schwarz-Pick matrix $\mathbf{P}_{n}^{b}\left(t_{0}\right)$ which is positive semidefinite since $b \in \mathcal{S}$.

Since the structured matrix $\mathbb{P}_{k}^{w}$ is Hermitan, it satisfies (see Theorem 7.1 in [6] for the proof) the Stein identity

$$
\mathbb{P}_{k}^{w}-T_{k} \mathbb{P}_{k}^{w} T_{k}^{*}=E_{k} E_{k}^{*}-M_{k} M_{k}^{*}
$$

where $T_{k}, E_{k}$ and $M_{k}$ are defined via formulas (2.4). Then it follows by Lemma 2.2 that the sequence $\left\{w_{0}, \ldots, w_{k}\right\}$ is $t_{0}$-isometric:

$$
\overline{\mathbb{U}_{k}^{w}} \mathbf{\Psi}_{k}\left(t_{0}\right) \mathbb{U}_{k}^{w}=\boldsymbol{\Psi}_{n}\left(t_{0}\right) .
$$

Consider the matrix

$$
\mathbb{P}_{k}^{s}:=\mathbb{H}_{k}^{s} \boldsymbol{\Psi}_{k}\left(t_{0}\right) \mathbb{U}_{k}^{s} .
$$

Upon substituting relations (2.16) into the right hand side expression and making use of (2.19) we get

$$
\begin{aligned}
\mathbb{P}_{k}^{s} & =\left(\left(\mathbb{U}_{k}^{f}\right)^{*} \mathbb{H}_{k}^{w}+\mathbb{H}_{k}^{f} \overline{\mathbb{U}_{k}^{w}}\right) \boldsymbol{\Psi}_{k}\left(t_{0}\right) \mathbb{U}_{k}^{w} \mathbb{U}_{k}^{f} \\
& =\left(\mathbb{U}_{k}^{f}\right)^{*} \mathbb{H}_{k}^{w} \boldsymbol{\Psi}_{n}\left(t_{0}\right) \mathbb{U}_{k}^{w} \mathbb{U}_{k}^{f}+\mathbb{H}_{k}^{f} \overline{\mathbb{U}^{w}} \mathbf{\Psi}_{n}\left(t_{0}\right) \mathbb{U}_{k}^{w} \mathbb{U}_{k}^{f} \\
& =\left(\mathbb{U}_{k}^{f}\right)^{*} \mathbb{H}_{k}^{w} \mathbf{\Psi}_{n}\left(t_{0}\right) \mathbb{U}_{k}^{w} \mathbb{U}_{k}^{f}+\mathbb{H}_{k}^{f} \boldsymbol{\Psi}_{n}\left(t_{0}\right) \mathbb{U}_{k}^{f}
\end{aligned}
$$

which on account of (2.15) and (2.18) can be written as

$$
\mathbb{P}_{k}^{s}=\left(\mathbb{U}_{k}^{f}\right)^{*} \mathbb{P}_{k}^{w} \mathbb{U}_{k}^{f}+\mathbb{P}_{k}^{f} .
$$

Since the matrices $\mathbb{P}_{k}^{w}$ and $\mathbb{P}_{k}^{f}$ are Hermitian, the matrix $\mathbb{P}_{k}^{s}$ is also Hermitian. It is readily seen from the structure of the matrices on the right hand side of (2.18) 
that the only entry in $\mathbb{P}_{k}^{f}$ that depends on $f_{2 k+1}$ is the lower diagonal entry

$$
\begin{aligned}
{\left[\mathbb{P}_{k}^{f}\right]_{k+1, k+1} } & =\sum_{\ell=0}^{k} \sum_{j=0}^{k-\ell} f_{k+\ell+1} \Psi_{\ell, k-j} f_{k-\ell-j}^{*} \\
& =(-1)^{k} t_{0}^{2 k+1} f_{2 k+1} f_{0}^{*}+\sum_{\ell=0}^{k-1} f_{k+\ell+1} \sum_{j=0}^{k-\ell} \Psi_{\ell, k-j} f_{k-\ell-j}^{*}
\end{aligned}
$$

which is nonnegative since $\mathbb{P}_{k}^{f} \geq 0$. Since $\left|f_{0}\right|=1$, we conclude that the replacement

$$
f_{2 k+1} \rightarrow f_{2 k+1}+(-1)^{k} \bar{t}_{0}^{2 k+1} f_{0} x \text { where } x>0
$$

increases the lower diagonal entry in $\mathbb{P}_{k}^{f}$ by $x$ and does not affect all the other entries. This replacement modifies $s_{2 k+1}$ accordingly to $(2.14)$

$$
s_{2 k+1} \rightarrow s_{2 k+1}+(-1)^{k} \bar{t}_{0}^{2 k+1} f_{0} w_{0} x=s_{2 k+1}+(-1)^{k} \bar{t}_{0}^{2 k+1} s_{0} x
$$

and does not change $s_{j}$ for $j \leq 2 k$. Since the matrix $\mathbb{U}_{k}^{f}$ depends only on $f_{0}, \ldots, f_{k}$, it follows from (2.20) that the entries in $\mathbb{P}_{k}^{s}$ do not change but the lower diagonal entry $\left[\mathbb{P}_{k}^{s}\right]_{k+1, k+1}$ which gets the same raise $x$ as $\left[\mathbb{P}_{k}^{f}\right]_{k+1, k+1}$. Thus, modifying $f_{2 k+1}$ as in $(2.21)$, one can increase the lower diagonal entries in $\mathbb{P}_{k}^{s}$ and $\mathbb{P}_{k}^{f}$ by any preassigned $x>0$. Since the $k \times k$ principal submatrices $\mathbb{P}_{k-1}^{s}$ and $\mathbb{P}_{k-1}^{f}$ in $(k+1) \times(k+1)$ matrices $\mathbb{P}_{k}^{s}$ and $\mathbb{P}_{k}^{f}$ are positive definite by the induction hypothesis, that latter modification makes $\mathbb{P}_{k}^{s}$ and $\mathbb{P}_{k}^{f}$ positive definite if $x$ is large enough. This completes the proof of the lemma.

Theorem 2.3 shows how one can generate infinite $t_{0}$-isometric sequences using unimodular functions analytic at $t_{0}$. Note that $t_{0}$-isometric sequences $\left\{w_{j}\right\}_{j=0}^{\infty}$ that arise in this way are characterized by the additional condition

$$
\limsup _{j \rightarrow \infty}\left|w_{j}\right|^{\frac{1}{j}}<\infty
$$

that guarantees a nonzero radius of convergence for the series in (2.6). The finite case is much simpler: the next theorem shows that every finite $t_{0}$-isometric sequence arises as a sequence of Taylor coefficients at $t_{0}$ of a ratio of two finite Blaschke products.

Theorem 2.5. Let $t_{0} \in \mathbb{T}$ and $w_{0}, \ldots, w_{2 n+1} \in \mathbb{C}$. The following are equivalent:

1. $\left|w_{0}\right|=1$ and the matrix $\mathbb{P}\left(t_{0} ; w_{0}, \ldots, w_{2 n+1}\right)$ defined in (1.13) is Hermitian.

2. The sequence $\left\{w_{0}, \ldots, w_{2 n+1}\right\}$ is $t_{0}$-isometric.

3. There exist finite Blaschke products $s$ and $f$ so that the function $w=\frac{s}{f}$ satisfies interpolation conditions

$$
w_{j}\left(t_{0}\right):=\frac{w^{(j)}\left(t_{0}\right)}{j !}=w_{j} \quad \text { for } j=0, \ldots, 2 n+1 .
$$


Proof. The implication (3) $\Rightarrow(2)$ follows by Statement 2 in Theorem 2.3.

$(2) \Rightarrow(1)$ : Let us assume that $\left\{w_{0}, \ldots, w_{2 n+1}\right\}$ is $t_{0}$-isometric and let the matrices $T_{2 n+1}, E_{2 n+1}$ and $M_{2 n+1}$ be defined via formulas (2.4). By Lemma 2.2, the Stein equation

$$
X-T_{2 n+1} X T_{2 n+1}^{*}=E_{2 n+1} E_{2 n+1}^{*}-M_{2 n+1} M_{2 n+1}^{*}
$$

has a solution $X=\left[X_{i j}\right]_{i, j=0}^{2 n+1}$. Comparing the corresponding entries in the equality (2.23) we come to the system

$$
\begin{aligned}
t_{0} X_{0 i}=\bar{t}_{0} X_{i 0} & =w_{i+1} w_{0}^{*} \quad(i=0, \ldots, 2 n), \\
t_{0} X_{i+1, j}+\bar{t}_{0} X_{i, j+1}+X_{i j} & =w_{i+1} w_{j+1}^{*}
\end{aligned} \quad(i=1, \ldots, 2 n ; j=0, \ldots, 2 n),
$$

which, upon being solved recursively, leads to

$$
X_{k \ell}=\sum_{j=0}^{\ell} \sum_{i=0}^{j}(-1)^{j}\left(\begin{array}{c}
j \\
i
\end{array}\right) t_{0}^{i+j+1} w_{k+i+1} w_{\ell-j}^{*}
$$

for

$$
0 \leq k+\ell \leq 2 n-1 \text {. }
$$

Thus, for every solution $X$ of the Stein equation (2.23), the entries with the indeces from the domain (2.25) are uniquely defined by the right hand side of the equation. Moreover, these entries satisfy the symmetry

$$
X_{k \ell}=X_{\ell k}^{*} \quad(0 \leq k+\ell \leq 2 n-1)
$$

which follows from the abovementioned uniqueness and the fact that $X^{*}$ satisfies the Stein equation (2.23) if and only if $X$ does. Making use of numbers $\Psi_{i j}$ given in (1.11), one can rewrite $(2.24)$ as

$$
X_{k \ell}=\sum_{j=0}^{\ell} \sum_{i=0}^{j} w_{k+i+1} \Psi_{i j} w_{\ell-j}^{*} \quad \text { for } \quad 0 \leq k+\ell \leq 2 n-1 .
$$

The latter means that for $k, \ell \in\{0, \ldots, n\}, X_{k \ell}$ is equal to the corresponding entry in the matrix $\mathbb{P}\left(t_{0} ; w_{0}, \ldots, w_{2 n+1}\right)$ defined by (1.13). In other words, $\mathbb{P}\left(t_{0} ; w_{0}, \ldots, w_{2 n+1}\right)$ is the $(n+1) \times(n+1)$ leading principal submatrix of $X$. It now follows from $(2.26)$ that $\mathbb{P}\left(t_{0} ; w_{0}, \ldots, w_{2 n+1}\right)$ is Hermitian. Equality $\left|w_{0}\right|=1$ is a part of the definition of $t_{0}$-symmetry.

$(1) \Rightarrow(3)$ : Let us assume that $\left|w_{0}\right|=1$ and the matrix $\mathbb{P}\left(t_{0} ; w_{0}, \ldots, w_{2 n+1}\right)$ defined in (1.13) is Hermitian. By Lemma 2.4, there exist $f_{0}, \ldots, f_{2 n+1} \in \mathbb{C}$ with $\left|f_{0}\right|=1$ such that the matrices $\mathbb{P}\left(t_{0} ; f_{0}, \ldots, f_{2 n+1}\right)$ and $\mathbb{P}\left(t_{0} ; s_{0}, \ldots, s_{2 n+1}\right)$ are positive definite where $s_{j}$ 's are the numbers given by (2.14). Since $s_{0}=w_{0} f_{0}$, we have $\left|s_{0}\right|=1$. By the interpolation result mentioned in the proof of Lemma 2.4, there exist finite Blaschke products $s(z)$ and $f(z)$ satisfying the conditions

$$
\frac{s^{(j)}\left(t_{0}\right)}{j !}=s_{j} \quad \text { and } \quad \frac{f^{(j)}\left(t_{0}\right)}{j !}=f_{j} \quad \text { for } j=0, \ldots, 2 n+1 .
$$


Now it follows from $(2.14)$ that the ratio $w(z)=\frac{s(z)}{f(z)}$ satisfies the conditions (2.22).

We conclude the section with several remarks. The first one (for the proof, we refer to [2, Corollary 7.9]) relates the third statements in Theorems 1.5 and 2.5:

Remark 2.6. Let $t_{0} \in \mathbb{T}, k \in \mathbb{Z}_{+}, w_{0}, \ldots, w_{k} \in \mathbb{C}$ and let $w$ be a function analytic in $\mathcal{U}_{t_{0}}$. Then the nontangential boundary limits $w_{j}\left(t_{0}\right)$ exist and satisfy

$$
w_{j}\left(t_{0}\right):=\lim _{z \rightarrow t_{0}} \frac{w^{(j)}(z)}{j !}=w_{j} \quad \text { for } \quad j=0, \ldots, k
$$

if and only if $w$ admits the asymptotic expansion

$$
w(z)=w_{0}+w_{1}\left(z-t_{0}\right)+\ldots+w_{k}\left(z-t_{0}\right)^{k}+o\left(\left(z-t_{0}\right)^{k} \quad \text { as } z \widehat{\rightarrow} t_{0} .\right.
$$

Remark 2.7. Note that any $t_{0}$-isometric sequence $\left\{w_{0}, \ldots, w_{n}\right\}$ admits a $t_{0}$-isometric extension.

Indeed, if $\left\{w_{0}, \ldots, w_{n}\right\}$ is $t_{0}$-isometric, then $\left|w_{0}\right|=1$ (by Remark 1.3) and the Stein equation (2.5) has a solution $X$ (by Lemma 2.2). It is easily seen that then $X^{*}$ also satisfies $(2.5)$ and therefore, $Y:=\frac{1}{2}\left(X+X^{*}\right)$ is a Hermitian solution of (2.5). By the second assertion in Lemma 2.2 , there exist $w_{n+1}, \ldots, w_{2 n+1}$ such that

$$
Y=\mathbb{P}\left(t_{0}, w_{0}, \ldots, w_{2 n+1}\right)
$$

and since the latter matrix is Hermitian, it follows by Theorem 2.5 that the sequence $\left\{w_{0}, \ldots, w_{2 n+1}\right\}$ is $t_{0}$-isometric.

\section{Proofs of Theorems 1.5 and 1.7}

To present the proofs of Theorems 1.5 and 1.7 we still need some preliminary results.

Lemma 3.1. Let $w$ be analytic on $\mathcal{U}_{t_{0}}$ and let $w^{(2 n+1)}$ be bounded on an open nontangential neighborhood $\Gamma_{t_{0}, \alpha}$ of $t_{0} \in \mathbb{T}$ :

$$
\left|w_{2 n+1}(z)\right| \leq \gamma \quad\left(z \in \Gamma_{t_{0}, \alpha}\right) .
$$

Then the following nontangential limits

$$
w_{j}\left(t_{0}\right)=\lim _{z \rightarrow t_{0}} w_{j}(z), \quad w_{j}(z):=\frac{w^{(j)}(z}{j !}
$$

exist for $j=0, \ldots, 2 n$ and

$$
w_{j}(z)=\sum_{i=0}^{2 n-j}\left(\begin{array}{c}
j+i \\
i
\end{array}\right) w_{j+i}\left(t_{0}\right)\left(z-t_{0}\right)^{i}+O\left(\left(z-t_{0}\right)^{2 n-j+1}\right) \quad\left(z \widehat{\rightarrow} t_{0}\right) .
$$


Proof. We start with the Taylor representation

$$
w_{j}(z)=\sum_{i=0}^{2 n-j} \frac{w_{j}^{(i)}(\omega)}{i !}(z-\omega)^{i}+\int_{\omega}^{z} \frac{w_{j}^{(2 n-j+1)}(\zeta)}{(2 n-j) !}(z-\zeta)^{2 n-j} d \zeta
$$

of the function $w_{j}(z)$ at a point $\omega \in \Gamma_{t_{0}, \alpha}$. Since

$$
\frac{w_{j}^{(i)}(z)}{i !}=\frac{w^{(j+i)}(z)}{i ! j !}=\frac{(j+i) !}{i ! j !} w_{j+i}(z)=\left(\begin{array}{c}
j+i \\
i
\end{array}\right) w_{j+i}(z),
$$

representation (3.3) can be written as

$$
\begin{aligned}
w_{j}(z)= & \sum_{i=0}^{2 n-j}\left(\begin{array}{c}
j+i \\
j
\end{array}\right) w_{j+i}(\omega)(z-\omega)^{i} \\
& +(2 n+1)\left(\begin{array}{c}
2 n \\
j
\end{array}\right) \int_{\omega}^{z} w_{2 n+1}(\zeta)(z-\zeta)^{2 n-j} d \zeta .
\end{aligned}
$$

The latter integral does not depend on the path of integration between $\omega$ and $z$. We set for short (we integrate along a rectifiable Jordan curve connecting $z$ and $\omega$ inside the unit disk $\mathbb{D}$ )

$$
G_{\omega, z}=\int_{\omega}^{z} w_{2 n+1}(\zeta)(z-\zeta)^{2 n-j} d \zeta
$$

Since $w(z)$ is bounded on $\Gamma_{t_{0}, \alpha}$, and $z-\zeta$ is also bounded on $\Gamma_{t_{0}, \alpha} \times \Gamma_{t_{0}, \alpha}$, we may conclude that the following two integrals are well defined and the following limit relations hold true

$$
G_{\omega, t_{0}}=\int_{\omega}^{t_{0}} w_{2 n+1}(\zeta)\left(t_{0}-\zeta\right)^{2 n-j} d \zeta=\lim _{z \rightarrow t_{0}} F_{\omega, z}
$$

and

$$
G_{t_{0}, z}=\int_{t_{0}}^{z} w_{2 n+1}(\zeta)(z-\zeta)^{2 n-j} d \zeta=\lim _{\omega \leftrightharpoons t_{0}} F_{\omega, z} .
$$

Sending $z \in \mathcal{U}$ to $t_{0}$ in (3.4) (for $\omega$ fixed) we conclude that there exists the limit

$$
w_{j}\left(t_{0}\right)=\lim _{z \rightarrow t_{0}} w_{j}(z)=\sum_{i=0}^{2 n-j}\left(\begin{array}{c}
j+i \\
j
\end{array}\right) w_{j+i}(\omega)\left(t_{0}-\omega\right)^{i}+(2 n+1)\left(\begin{array}{c}
2 n \\
j
\end{array}\right) G_{\omega, t_{0}}
$$

for $j=0, \ldots, 2 n$. This proves the first assertion of the lemma and allows us to pass to limits in (3.4) as $\omega \in \Gamma_{t_{0}, \alpha}$ tends to $t_{0}$ for a fixed $z$ to get

$$
w_{j}(z)=\sum_{i=0}^{2 n-j}\left(\begin{array}{c}
j+i \\
j
\end{array}\right) w_{j+i}\left(t_{0}\right)\left(z-t_{0}\right)^{i}+(2 n+1)\left(\begin{array}{c}
2 n \\
j
\end{array}\right) G_{t_{0}, z} .
$$

Upon choosing the path of integration for $G_{t_{0}, z}$ to be the line segment connecting the points $z$ and $t_{0}$ and making use of (3.1), we get the estimate

$$
\left|G_{t_{0}, z}\right| \leq \gamma\left|z-t_{0}\right|^{2 n-j+1}
$$

which implies (3.2). 
In the next lemma,

$$
\mathbf{P}_{i, j}^{w}(z)=\frac{1}{i ! j !} \frac{\partial^{i+j}}{\partial z^{i} \partial \bar{z}^{j}} \frac{1-|w(z)|^{2}}{1-|z|^{2}}
$$

stands for the $i j$-th entry of the Schwarz-Pick matrix $\mathbf{P}_{n}^{w}(z)$.

Lemma 3.2. Let $w(z)$ and $f(z)$ be two functions analytic on $\mathbb{D}$ such that $w^{(2 n+1)}(z)$ and $f^{(2 n+1)}(z)$ are bounded in some open nontangential neighborhood $\Gamma_{t_{0}, \alpha}$ of $t_{0} \in$ $\mathbb{T}$ and let

$$
\lim _{z \rightarrow t_{0}} w_{j}(z)=\lim _{z \rightarrow t_{0}} f_{j}(z)=: w_{j}\left(t_{0}\right) \quad \text { for } \quad j=0, \ldots, 2 n
$$

(note that the existence of limits in (3.8) is guaranteed by Lemma 3.1). Then

$$
\mathbf{P}_{i, j}^{w}(z)-\mathbf{P}_{i, j}^{f}(z)=O\left(\left(z-t_{0}\right)^{2 n-i-j}\right)
$$

for $i, j=0, \ldots, n$ as $z \widehat{\rightarrow} t_{0}$. In particular, $d_{w, n}(z)-d_{f, n}(z)=O(1)$.

Proof. Straightforward differentiation gives

$$
\frac{1}{i ! j !} \frac{\partial^{i+j}}{\partial z^{i} \partial \bar{z}^{j}} \frac{|w(z)|^{2}}{1-|z|^{2}}=\sum_{k=0}^{i} \sum_{\ell=0}^{j} w_{i-k}(z) \frac{u_{k, \ell}(z)}{\left(1-|z|^{2}\right)^{k+\ell+1}} w_{j-\ell}(z)^{*},
$$

where, as before, $w_{j}(z)$ stands for $\frac{1}{j !} w^{(j)}(z)$ and where

$$
u_{k, \ell}(z)=\sum_{m=0}^{\min (k, \ell)} \frac{(k+\ell-m) !}{(k-m) !(\ell-m) ! m !} \bar{z}^{k-m} z^{\ell-m}\left(1-|z|^{2}\right)^{m}, .
$$

Substituting (3.7), (3.10) and similar formulas for $f$ into (3.9) we get

$$
\begin{aligned}
\mathbf{P}_{i, j}^{w}(z)-\mathbf{P}_{i, j}^{f}(z)= & \frac{1}{i ! j !} \frac{\partial^{i+j}}{\partial z^{i} \partial \bar{z}^{j}} \frac{|f(z)|^{2}-|w(z)|^{2}}{1-|z|^{2}} \\
= & \sum_{k=0}^{i} \sum_{\ell=0}^{j} f_{i-k}(z) \frac{u_{k, \ell}(z)}{\left(1-|z|^{2}\right)^{k+\ell+1}} f_{j-\ell}(z)^{*} \\
& -\sum_{k=0}^{i} \sum_{\ell=0}^{j} w_{i-k}(z) \frac{u_{k, \ell}(z)}{\left(1-|z|^{2}\right)^{k+\ell+1}} w_{j-\ell}(z)^{*} \\
= & \sum_{k=0}^{i} \sum_{\ell=0}^{j}\left(f_{i-k}(z)-w_{i-k}(z)\right) \frac{u_{k, \ell}(z) f_{j-\ell}(z)^{*}}{\left(1-|z|^{2}\right)^{k+\ell+1}} \\
& +\sum_{k=0}^{i} \sum_{\ell=0}^{j} \frac{w_{i-k}(z) u_{k, \ell}(z)}{\left(1-|z|^{2}\right)^{k+\ell+1}}\left(f_{j-\ell}(z)^{*}-w_{j-\ell}(z)^{*}\right) .
\end{aligned}
$$

Applying (3.2) to the functions $w_{k}$ and $f_{k}$ and making use of equalities (3.8), we conclude that

$$
w_{k}(z)-f_{k}(z)=O\left(\left(z-t_{0}\right)^{2 n-k+1}\right) \quad \text { as } z \widehat{\rightarrow} t_{0}
$$


for $k=0, \ldots, 2 n$. Since $z-t_{0}=O\left(1-|z|^{2}\right)$ when $z \widehat{\rightarrow} t_{0}$ nontangentially, we have

$$
\begin{aligned}
& \left.\frac{f_{i-k}(z)-w_{i-k}(z)}{\left(1-|z|^{2}\right)^{k+\ell+1}}=O\left(\left(z-t_{0}\right)^{2 n-i-\ell}\right)\right), \quad \leq k \leq i \leq n, \\
& \frac{f_{j-\ell}(z)-w_{j-\ell}(z)}{\left(1-|z|^{2}\right)^{k+\ell+1}}=O\left(\left(z-t_{0}\right)^{2 n-j-k}\right), \quad 0 \leq \ell \leq j \leq n .
\end{aligned}
$$

Now asymptotic relation (3.9) follows from (3.12), (3.13) and (3.14). The last assertion follows from (3.9) upon letting $i=j=n$.

Lemma 3.3. Let $w$ and $f$ be two functions analytic on a neighborhood $\mathcal{U}_{t_{0}}$ of $t_{0} \in \mathbb{T}$ and let us assume that the nontangential boundary limits of their $2 n+2$ first derivatives at $t_{0}$ exist and are equal:

$$
w_{j}\left(t_{0}\right)=f_{j}\left(t_{0}\right) \quad \text { for } j=0, \ldots, 2 n+1 .
$$

Then

$$
\mathbf{P}_{n}^{w}(z)-\mathbf{P}_{n}^{f}(z)=o(1) \quad \text { as } \quad \widehat{\rightarrow} t_{0}
$$

where $\mathbf{P}_{n}^{w}(z)$ and $\mathbf{P}_{n}^{f}(z)$ are the Schwarz-Pick matrices associated with $w$ and $f$ via formula (1.3).

Proof. By Remark 2.6, equalities (3.15) are equivalent to the asymptotic relation

$$
w(z)-f(z)=o\left(\left(z-t_{0}\right)^{2 n+1} \quad \text { as } z \widehat{\rightarrow} t_{0} .\right.
$$

Therefore we have

$$
w_{k}(z)-f_{k}(z)=o\left(\left(z-t_{0}\right)^{2 n-k+1}\right) \quad \text { for } k=1, \ldots, 2 n+1 \text { as } z \rightarrow t_{0} .
$$

Substituting the latter asymptotics into (3.9) we get

$$
\mathbf{P}_{i, j}^{w}(z)-\mathbf{P}_{i, j}^{f}(z)=o\left(\left(z-t_{0}\right)^{2 n-i-j}\right) \quad(i, j=0, \ldots, n)
$$

which clearly imply (3.16).

Proof of Theorem 1.5: The third statement in Theorem 1.5 can be reformulated equivalently: there exists a rational unimodular function $f$ satisfying conditions (3.15). Now we apply Theorem 2.5 to the sequence $\left\{w_{0}, \ldots, w_{2 n+1}\right\}$ where $w_{j}:=$ $w_{j}\left(t_{0}\right)$ to conclude that the Statements 1-3 in Theorem 1.5 are equivalent.

Assume that the asymptotic equality (1.18) holds (or equivalently, the equalities (3.15) hold) for a rational unimodular function $f$. Then $\mathbb{P}_{n}^{w}\left(t_{0}\right)=\mathbb{P}_{n}^{f}\left(t_{0}\right)$, by the definition (1.9), and the relation (3.16) holds by Lemma 3.3. Furthermore, since $f$ is rational and unimodular on $\mathbb{T}$, the boundary Schwarz-Pick matrix $\mathbf{P}_{n}^{f}\left(t_{0}\right)$ exists and is equal to $\mathbb{P}_{n}^{f}\left(t_{0}\right)$, by Statement 1 in Theorem 2.3. Now we pass to nontangential limits in (3.16) to get

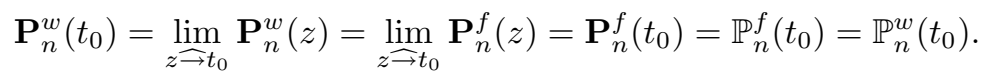

To complete the proof of Theorem 1.5, it suffices to show that (3) $\Rightarrow(4) \Rightarrow(2)$. 
Proof of $(3) \Rightarrow(4)$ : Assume that the asymptotic equality

$$
w(z)=f(z)+o\left(\left(z-t_{0}\right)^{2 n+1}\right)
$$

holds (or equivalently, the equalities (3.15) hold) for a rational unimodular function $f$ as $z \widehat{\rightarrow} t_{0}$ from inside of the disk $\mathbb{D}$. Then we also have for $|z|>1$,

$$
w(z):=\overline{w(1 / \bar{z})}^{-1}=\overline{f(1 / \bar{z})}^{-1}+{\overline{o\left(\left(\frac{1}{\bar{z}}-t_{0}\right)^{2 n+1}\right)}}^{=} f(z)+o\left(\left(z-t_{0}\right)^{2 n+1}\right)
$$

as $z \widehat{\rightarrow} t_{0}$ from outside of $\mathbb{D}$, where $f(z)$ stands for both symmetry and analytic continuation, that agree since $f$ is rational and unimodular on $\mathbb{T}$. Since $f$ is analytic in a neighborhood of $t_{0}$, its asymptotics form inside and outside of the unit disk agree. The asymptotic equality (1.19) follows from the fact that the limits $w_{j}\left(t_{0}\right)$ exist for $j=0, \ldots, n$ as $z \widehat{\rightarrow} t_{0}$ from inside and outside of $\mathbb{D}$ (by Remark 2.6) and are equal.

Proof of $(4) \Rightarrow(2)$ : Let us assume that the asymptotic relations (1.19) hold from inside and outside of $\mathbb{D}$. By definition of symmetry continuation we have

$$
\overline{w(1 / \bar{z})} \equiv w(z)^{-1}, \quad|z|<1 .
$$

Since

$$
\frac{1}{z}-\bar{t}_{0}=\frac{-\bar{t}_{0}\left(z-t_{0}\right)}{z}=O\left(\left(z-t_{0}\right)\right) \quad \text { as } z \rightarrow t_{0},
$$

it follows, by substituting $1 / \bar{z},|z|<1$ in (1.19), that

$$
\overline{w(1 / \bar{z})}=\sum_{j=0}^{k} w_{j}\left(t_{0}\right)^{*}\left(\frac{1}{z}-\bar{t}_{0}\right)^{j}+o\left(\left(z-t_{0}\right)^{k}\right)
$$

for $k=0, \ldots, 2 n+1$ and therefore, that

$$
\begin{aligned}
z^{k} w(z)^{-1} \equiv z^{k} \overline{w(1 / \bar{z})} & =\sum_{j=0}^{k} w_{j}\left(t_{0}\right)^{*} z^{k-j}\left(1-z \overline{t_{0}}\right)^{j}+o\left(\left(z-t_{0}\right)^{k}\right) \\
& =\sum_{j=0}^{k}\left(-\bar{t}_{0}\right)^{j} w_{j}\left(t_{0}\right)^{*} z^{k-j}\left(z-t_{0}\right)^{j}+o\left(\left(z-t_{0}\right)^{k}\right) .
\end{aligned}
$$

for $|z|<1$. Simple rearrangements based on the relation $z^{\ell}=\sum_{i=0}^{\ell}\left(\begin{array}{c}\ell \\ i\end{array}\right) t_{0}^{\ell-i}\left(z-t_{0}\right)^{i}$, lead us to

$$
z^{k} w(z)^{-1}=\sum_{j=0}^{k}\left(\sum_{\ell=0}^{k-j}(-1)^{\ell} t_{0}^{j-\ell}\left(\begin{array}{c}
k-\ell \\
j
\end{array}\right) w_{\ell}\left(t_{0}\right)^{*}\right)\left(z-t_{0}\right)^{k-j}+o\left(\left(z-t_{0}\right)^{k}\right) .
$$


Upon multiplying the corresponding parts both in the latter equality and (1.19) (for $|z|<1$ ) we get

$$
\begin{aligned}
& \left(\sum_{j=0}^{2 n+1} w_{j}\left(t_{0}\right)\left(z-t_{0}\right)^{j}\right)\left(\sum_{j=0}^{k}\left(\sum_{\ell=0}^{k-j}(-1)^{\ell} t_{0}^{j-\ell}\left(\begin{array}{c}
k-\ell \\
j
\end{array}\right) w_{\ell}\left(t_{0}\right)^{*}\right)\left(z-t_{0}\right)^{k-j}\right) \\
& =z^{k}+o\left(\left(z-t_{0}\right)^{k}\right)=\sum_{j=0}^{k}\left(\begin{array}{c}
k \\
j
\end{array}\right) t_{0}^{k-j}\left(z-t_{0}\right)^{j}+o\left(\left(z-t_{0}\right)^{k}\right)
\end{aligned}
$$

Comparing the coefficients of $\left(z-t_{0}\right)^{k}$ in the latter equality we eventually arrive at

$$
\sum_{j=0}^{k} \sum_{\ell=0}^{k-j}(-1)^{\ell}\left(\begin{array}{c}
k-\ell \\
j
\end{array}\right) t_{0}^{j-\ell} w_{j}\left(t_{0}\right) w_{\ell}\left(t_{0}\right)^{*}=1 \quad \text { for } k=0, \ldots, 2 n+1 .
$$

By Remark 2.1, the latter equalities mean that the sequence $\left\{w_{0}\left(t_{0}\right), \ldots, w_{2 n+1}\left(t_{0}\right)\right\}$ is $t_{0}$-isometric.

Proof of Theorem 1.7: Let us assume that $w^{(2 n+1)}$ is bounded on $\Gamma_{t_{0}, \alpha}$ and that the sequence $\left\{w_{0}, \ldots, w_{2 n}\right\}$ of the nontangential boundary limits $w_{j}:=w_{j}\left(t_{0}\right)$ is $t_{0}$-isometric (the existence of the latter limits is guaranteed by Lemma 3.1). Let $w_{2 n+1}$ be any number such that the extended sequence $\left\{w_{0}, \ldots, w_{2 n}, w_{2 n+1}\right\}$ is $t_{0}$-isometric (such a number exists by Remark 2.7). By Theorem 2.5, there exists a rational unimodular function $f$ such that $f_{j}\left(t_{0}\right)=w_{j}$ for $j=0, \ldots, 2 n+1$. The functions $f$ and $w$ meets all the conditions in Lemma 3.2. Therefore,

$$
d_{w, n}(z)-d_{f, n}(z)=O(1)
$$

as $z$ tends to $t_{0}$ nontangentially. Since $f$ is rational unimodular function the limit $d_{f, n}\left(t_{0}\right)$ exists and in particular, $d_{f, n}(z)$ is bounded on $\Gamma_{t_{0}, \alpha}$. Then (3.20) implies $(1.21)$. Equivalences $(1) \Leftrightarrow(2)) \Leftrightarrow(3)$ in Theorem 1.7 are established in much the same way as those in Theorem 1.5.

\section{Generalized Schur functions}

Condition (1.15) does not make much sense for general analytic functions. For example, the function $w(z)=e^{\frac{i}{1-z}}$ meets condition (1.15) (for $n=0$ ) at $t_{0}=1$, since $|w(z)|=1$ on the radius $[0,1)$; however, it has no radial limit as $z$ approaches 1 . Thus the extension of Theorem 1.4 (at least in its present formulation) to general analytic functions is unlike. However, this theorem can be extended to some classes of functions that are close in some sense to $\mathcal{S}$. In this section we will show how it can be done for the so-called generalized Schur functions. Recall that a function $w$ belongs to the generalized Schur class $\mathcal{S}_{\kappa}$ if it admits a representation of the form

$$
w(z)=\frac{s(z)}{b(z)},
$$


where $s$ is a Schur class function and $b$ is a finite Blaschke product $b$ of degree $\kappa$, having disjoint zeroes in $\mathbb{D}$. A well known property of $\mathcal{S}_{\kappa}$ functions is that the kernel

$$
K_{w}(z, \zeta):=\frac{1-w(z) \overline{w(\zeta)}}{1-z \bar{\zeta}}
$$

has $\kappa$ negative squares on $\rho(w)$, the domain of analyticity of $w$ intersected with $\mathbb{D}$. Therefore, the Schwarz-Pick matrix $\mathbf{P}_{n}^{w}(z)$ defined in (1.3) makes sense and has not more than $\kappa$ negative eigenvalues (counted with multiplicities) for every $z \in \rho(w)$ and every $n \in \mathbb{Z}_{+} ;$in formulas: sq_$_{-} \mathbf{P}_{n}^{w}(z) \leq \kappa$.

Remark 4.1. It is that once the boundary Schwarz-Pick matrix $\mathbf{P}_{n}^{w}\left(t_{0}\right)$ exists, sq_ $\mathbf{P}_{n}^{w}\left(t_{0}\right) \leq \kappa$.

The next theorem extends Theorem 1.4.

Theorem 4.2. Let $w \in \mathcal{S}_{\kappa}, t_{0} \in \mathbb{T}, n \in \mathbb{Z}_{+}$and let $d_{w, n}$ be defined as in (1.5). The following are equivalent.

1. $\widetilde{d}:=\liminf _{z \rightarrow t_{0}} d_{w, n}(z)<\infty$.

2. $d_{w, n}\left(t_{0}\right):=\lim _{z \rightarrow t_{0}} d_{w, n}(z)<\infty$.

3. The boundary Schwarz-Pick matrix $\mathbf{P}_{n}^{w}\left(t_{0}\right)$ exists.

4. The nontangential boundary limits $w_{j}\left(t_{0}\right)$ exist for $j=0, \ldots, 2 n+1$ and satisfy

$$
\left|w_{0}\left(t_{0}\right)\right|=1, \quad \mathbb{P}_{n}^{w}\left(t_{0}\right)=\mathbb{P}_{n}^{w}\left(t_{0}\right)^{*} \quad \text { and } \quad \mathrm{sq}_{-} \mathbb{P}_{n}^{w}\left(t_{0}\right) \leq \kappa,
$$

where $\mathbb{P}_{n}^{w}\left(t_{0}\right)$ is the matrix defined in (1.9).

Moreover, when these conditions hold, then

$$
\tilde{d}=d_{w, n}\left(t_{0}\right) \quad \text { and } \quad \mathbf{P}_{n}^{w}\left(t_{0}\right)=\mathbb{P}_{n}^{w}\left(t_{0}\right) .
$$

For $w$ of the form (4.1) we have

$$
\frac{1-|w(z)|^{2}}{1-|z|^{2}}=\frac{1-|s(z)|^{2}}{|b(z)|^{2}\left(1-|z|^{2}\right)}-\frac{1-|b(z)|^{2}}{|b(z)|^{2}\left(1-|z|^{2}\right)} .
$$

Upon applying $\frac{1}{i ! j !^{2}} \frac{\partial^{i+j}}{\partial z^{i} \partial j^{n}}$ to both parts of the latter equality and arranging the obtained $(n+1)^{2}$ equalities in the matrix form, we get, by the definitions (1.3),

$$
\mathbf{P}_{n}^{w}(z)=L^{s, b}(z)-L^{b, b}(z),
$$

where we have set

$$
\begin{aligned}
L^{s, b}(z) & :=\left[\frac{1}{i ! j !} \frac{\partial^{i+j}}{\partial z^{i} \partial \bar{z}^{j}} \frac{1}{b(z)} \frac{1-|s(z)|^{2}}{1-|z|^{2}} \frac{1}{\overline{b(z)}}\right]_{i, j=0}^{n}, \\
L^{b, b}(z) & :=\left[\frac{1}{i ! j !} \frac{\partial^{i+j}}{\partial z^{i} \partial \bar{z}^{j}} \frac{1}{b(z)} \frac{1-|b(z)|^{2}}{1-|z|^{2}} \frac{1}{\overline{b(z)}}\right]_{i, j=0}^{n} .
\end{aligned}
$$


Note that if $K$ is a sesqui-analytic kernel and $f$ is analytic at $z$, then the equality

$$
\left[\frac{1}{i ! j !} \frac{\partial^{i+j}}{\partial z^{i} \partial \bar{z}^{j}} f(z) K(z, z) \overline{f(z)}\right]_{i, j=0}^{n}=\mathbb{U}_{n}^{f}(z)^{*} \cdot\left[\frac{1}{i ! j !} \frac{\partial^{i+j}}{\partial z^{i} \partial \bar{z}^{j}} K(z, z)\right]_{i, j=0}^{n} \cdot \mathbb{U}_{n}^{f}(z)
$$

holds by the Leibnitz rule. Upon applying this equality to

$$
K(z, \zeta)=\frac{1-s(z) \overline{s(\zeta)}}{1-z \bar{\zeta}} \text { and } f(z)=\frac{1}{b(z)}
$$

and taking into account that $\mathbb{U}_{n}^{1 / b}(z)=\mathbb{U}_{n}^{b}(z)^{-1}$ whenever $b(z) \neq 0$, we get

$$
L^{s, b}(z):=\mathbb{U}_{n}^{b}(z)^{-*} \mathbf{P}_{n}^{s}(z) \mathbb{U}_{n}^{b}(z)^{-1}
$$

where $\mathbf{P}_{n}^{s}(z)$ is the Schwarz-Pick matrix defined via formula (1.3). Letting $s=b$ in the latter formula, we get

$$
L^{b, b}(z)=\mathbb{U}_{n}^{b}(z)^{-*} \mathbf{P}_{n}^{b}(z) \mathbb{U}_{n}^{b}(z)^{-1} .
$$

Remark 4.3. Let $s$ be a Schur function, let $b$ be a finite Blaschke product and let $L^{s, b}(z)$ and $L^{b, b}(z)$ be defined as in (4.4) and (4.5). Then

1. The nontangential limit

$$
L^{s, b}\left(t_{0}\right):=\lim _{z \widehat{\rightarrow} t_{0}} L^{s, b}(z)
$$

exists if and only if the boundary Schwarz-Pick matrix $\mathbf{P}_{n}^{s}\left(t_{0}\right)$ exists and in this case

$$
L^{s, b}\left(t_{0}\right)=\mathbb{U}_{n}^{b}\left(t_{0}\right)^{-*} \mathbf{P}_{n}^{s}\left(t_{0}\right) \mathbb{U}_{n}^{b}\left(t_{0}\right)^{-1} .
$$

2. The limit

$$
L^{b, b}\left(t_{0}\right)=\lim _{z \rightarrow t_{0}} L^{b, b}(z)=\mathbb{U}_{n}^{b}\left(t_{0}\right)^{-*} \mathbf{P}_{n}^{b}\left(t_{0}\right) \mathbb{U}_{n}^{b}\left(t_{0}\right)^{-1}
$$

exists for every $n \in \mathbb{Z}_{+}$, when $z$ tends to $t_{0} \in \mathbb{T}$ unrestrictedly in $\mathbb{C}$.

Proof. Since $b$ is analytic at $t_{0} \in \mathbb{T}$ and $b\left(t_{0}\right) \neq 0$, the limit matrix $\mathbb{U}_{n}^{b}\left(t_{0}\right):=$ $\lim _{z \rightarrow t_{0}} \mathbb{U}_{n}^{b}(z)$ exists and is invertible. Now the first assertion follows from (4.6) upon passing to limits as $z \widehat{\rightarrow} t_{0}$. The second assertion follows since $\mathbf{P}_{n}^{b}\left(t_{0}\right)$ exists, by Theorem 2.3.

Corollary 4.4. Let $w \in \mathcal{S}_{\kappa}$ be of the form (4.1) with a Schur function $s$ and a finite Blaschke product $b$. Then the boundary Schwarz-Pick matrix $\mathbf{P}_{n}^{w}\left(t_{0}\right)$ exists if and only if $\mathbf{P}_{n}^{s}\left(t_{0}\right)$ exists and in this case,

$$
\mathbf{P}_{n}^{w}\left(t_{0}\right)=\mathbb{U}_{n}^{b}\left(t_{0}\right)^{-*}\left(\mathbf{P}_{n}^{s}\left(t_{0}\right)-\mathbf{P}_{n}^{b}\left(t_{0}\right)\right) \mathbb{U}_{n}^{b}\left(t_{0}\right)^{-1} .
$$

Proof. Since the limit (4.10) exists no matter how $z$ tends to $t_{0}$, it follows from (4.3) that $\mathbf{P}_{n}^{w}\left(t_{0}\right)$ exists if and only $L^{s, b}\left(t_{0}\right)$ exists; the latter is equivalent to the existence of $\mathbf{P}_{n}^{s}\left(t_{0}\right)$, by Remark 4.3. Passing to the limits in (4.3) as $z \widehat{\rightarrow} t_{0}$ and making use of (4.9) and (4.10) we arrive at (4.11). 
Lemma 4.5. Let $s \in \mathcal{S}_{0}$ be a Schur function, let $f$ be a function analytic at $t_{0} \in \mathbb{T}$ and let $n \in \mathbb{Z}_{+}$. Then

$$
\liminf _{z \rightarrow t_{0}} \frac{\partial^{2 n}}{\partial z^{n} \partial \bar{z}^{n}}\left(f(z) \frac{1-|s(z)|^{2}}{1-|z|^{2}} \overline{f(z)}\right)<\infty
$$

if and only if (the "if" part is obvious) the following limits exist and are finite:

$$
\lim _{z \rightarrow t_{0}} \frac{\partial^{i+j}}{\partial z^{i} \partial \bar{z}^{j}}\left(f(z) \frac{1-|s(z)|^{2}}{1-|z|^{2}} \overline{f(z)}\right)<\infty \quad(i, j=0, \ldots, n) .
$$

Furthermore,

$$
\liminf _{z \rightarrow t_{0}} \frac{\partial^{2 n}}{\partial z^{n} \partial \bar{z}^{n}}\left(f(z) \frac{1-|s(z)|^{2}}{1-|z|^{2}} \overline{f(z)}\right)=\lim _{z \widehat{\rightarrow} t_{0}} \frac{\partial^{2 n}}{\partial z^{n} \partial \bar{z}^{n}}\left(f(z) \frac{1-|s(z)|^{2}}{1-|z|^{2}} \overline{f(z)}\right) .
$$

The above result has been proved in [5] in the context of operator-valued Schur functions $s$ and vector valued functions $f$. The result is useful even in the scalarvalued setting due to the following

Corollary 4.6. Let $s$ be a Schur function, let $b$ be a finite Blaschke product and let

$$
L_{n n}^{s, b}(z)=\frac{1}{(n !)^{2}} \frac{\partial^{2 n}}{\partial z^{n} \partial \bar{z}^{n}} \frac{1-|s(z)|^{2}}{|b(z)|^{2}\left(1-|z|^{2}\right)},
$$

the lower diagonal element of the matrix $L^{s, b}(z)$ given in (4.4) be subject to

$$
\liminf _{z \rightarrow t_{0}} L_{n n}^{s, b}(z)<\infty \quad\left(t_{0} \in \mathbb{T}\right) .
$$

Then the nontangential boundary limit (4.8) exists and

$$
\lim _{z \widehat{\rightarrow} t_{0}} L_{n n}^{s, b}(z)=\liminf _{z \rightarrow t_{0}} L_{n n}^{s, b}(z) .
$$

Furthermore, the boundary Schwarz-Pick matrix $\mathbf{P}_{n}^{s}\left(t_{0}\right)$ exists.

Proof. Condition means that we can apply Lemma 4.5 for $f(z)=\frac{1}{b(z)}$. Then relations (4.12) mean that all the entries in the matrix $L^{s, b}(z)$ possess nontangential boundary limits and thus, the limit (4.8) exists. Then $\mathbf{P}_{n}^{s}\left(t_{0}\right)$ exists by Remark 4.3. Finally, equality (4.13) with $f=\frac{1}{b}$ gives (4.16).

Proof of Theorem 4.2: Let $w \in \mathcal{S}_{\kappa}$ be a generalized Schur function with the KreinLanger representation (4.1) and let us assume that

$$
\widetilde{d}:=\liminf _{z \rightarrow t_{0}} d_{w, n}(z)<\infty
$$

Equating the lower diagonal entries in the matrix identity (4.3) gives

$$
d_{w, n}(z)=L_{n n}^{s, b}(z)-L_{n n}^{b}(z)
$$


where $L_{n n}^{s, b}(z)$ is given in (4.14) and where, according to (4.5)

$$
L_{n n}^{b}(z)=\frac{1}{(n !)^{2}} \frac{\partial^{2 n}}{\partial z^{n} \partial \bar{z}^{n}} \frac{1-|b(z)|^{2}}{|b(z)|^{2}\left(1-|z|^{2}\right)} .
$$

By Remark 4.3, the limit

$$
L_{n n}^{b}\left(t_{0}\right)=\lim _{z \rightarrow t_{0}} L_{n n}^{b}(z)
$$

exists. Then it follows from (4.17) that

$$
\liminf _{z \rightarrow t_{0}} d_{w, n}(z)=\liminf _{z \rightarrow t_{0}} L_{n n}^{s, b}(z)-L_{n n}^{b}\left(t_{0}\right)
$$

and since the limit inferior on the left is finite, we conclude that condition (4.15) is satisfied. Then the boundary Schwarz-Pick matrix $\mathbf{P}_{n}^{s}\left(t_{0}\right)$ exists by Corollary 4.6. By Corollary 4.4, the boundary Schwarz-Pick matrix $\mathbf{P}_{n}^{w}\left(t_{0}\right)$ also exists. Now we can pass to the limits in (4.17) as $z \widehat{\rightarrow} t_{0}$ :

$$
d_{w, n}\left(t_{0}\right)=\lim _{z \rightarrow t_{0}} d_{w, n}(z)=\lim _{z \rightarrow t_{0}} L_{n n}^{s, b}(z)-L_{n n}^{b}\left(t_{0}\right)
$$

which implies, on account of (4.18) and of equality (4.16) (that holds, by Corollary 4.6)

$$
d_{w, n}\left(t_{0}\right)=\liminf _{z \rightarrow t_{0}} d_{w, n}(z) .
$$

This completes the proof of implications $(1) \Rightarrow(3) \Rightarrow(2)$ in Theorem 4.2 and also the first equality in (4.2).

Furthermore, since $s \in \mathcal{S}$, the existence of $\mathbf{P}_{n}^{s}\left(t_{0}\right)$ guarantees (by Theorem 1.4) the existence of the nontangential boundary limits

$$
s_{j}\left(t_{0}\right):=\lim _{z \rightarrow t_{0}} \frac{s^{(j)}(z)}{j !} \text { for } j=0, \ldots, 2 n+1
$$

such that $\left|s_{0}\left(t_{0}\right)\right|=1$ and the matrix $\mathbb{P}_{n}^{s}\left(t_{0}\right)$ defined via formula (1.9) is Hermitian. Then the sequence $\left\{s_{0}\left(t_{0}\right), \ldots, s_{2 n+1}\left(t_{0}\right)\right\}$ is $t_{0}$-isometric, by Theorem 2.5:

$$
\overline{\mathbb{U}}_{2 n+1}^{s}\left(t_{0}\right) \boldsymbol{\Psi}_{2 n+1}\left(t_{0}\right) \mathbb{U}_{2 n+1}^{s}\left(t_{0}\right)=\boldsymbol{\Psi}_{2 n+1}\left(t_{0}\right) .
$$

The sequence $\left\{b_{0}\left(t_{0}\right), \ldots, b_{2 n+1}\left(t_{0}\right)\right\}$ is $t_{0}$-isometric, by the second assertion in Theorem 2.3: Thus, we have

$$
\overline{\mathbb{U}}_{2 n+1}^{b}\left(t_{0}\right) \boldsymbol{\Psi}_{2 n+1}\left(t_{0}\right) \mathbb{U}_{2 n+1}^{b}\left(t_{0}\right)=\boldsymbol{\Psi}_{2 n+1}\left(t_{0}\right) .
$$

Since $b$ is analytic at $t_{0}$ and since $b\left(t_{0}\right) \neq 0$, the existence of the boundary limits

$$
w_{j}\left(t_{0}\right):=\lim _{z \rightarrow t_{0}} \frac{w^{(j)}(z)}{j !} \quad(j=0, \ldots, 2 n+1)
$$

follows from (4.1) and (4.21). Since $s=w b$, we have $\mathbb{U}_{2 n+1}^{s}\left(t_{0}\right)=\mathbb{U}_{2 n+1}^{w}\left(t_{0}\right) \mathbb{U}_{2 n+1}^{b}\left(t_{0}\right)$ so that

$$
\mathbb{U}_{2 n+1}^{w}\left(t_{0}\right)=\mathbb{U}_{2 n+1}^{s}\left(t_{0}\right) \mathbb{U}_{2 n+1}^{b}\left(t_{0}\right)^{-1} .
$$

The latter equality together with (4.20) and (4.21) implies

$$
\overline{\mathbb{U}}_{2 n+1}^{w}\left(t_{0}\right) \mathbf{\Psi}_{2 n+1}\left(t_{0}\right) \mathbb{U}_{2 n+1}^{w}\left(t_{0}\right)=\boldsymbol{\Psi}_{2 n+1}\left(t_{0}\right)
$$


which means that the sequence $\left\{w_{0}\left(t_{0}\right), \ldots, w_{2 n+1}\left(t_{0}\right)\right\}$ is $t_{0}$-isometric. By Theorem 1.5, conditions (1.17) are satisfied and the matrix $\mathbb{P}_{n}^{w}\left(t_{0}\right)$ constructed from the limits (4.22) via formula (1.9), is equal to the boundary Schwarz-Pick matrix $\mathbf{P}_{n}^{w}\left(t_{0}\right)$. This proves the second equality in (4.2). Furthermore, this equality implies that $\mathbb{P}_{n}^{w}\left(t_{0}\right)$ is Hermitian and (by Remark 4.1) that sq_ $\mathbf{P}_{n}^{w}\left(t_{0}\right) \leq \kappa$. This completes the proof of Theorem 4.2.

The next theorem partly generalizes Theorem 4.2.

Theorem 4.7. Let $w=\frac{s}{f}$ be the ratio of two Schur class functions satisfying conditions

$$
\liminf _{z \rightarrow t_{0}} d_{s, n}(z)<\infty \quad \text { and } \quad \liminf _{z \rightarrow t_{0}} d_{f, n}(z)<\infty
$$

where $t_{0} \in \mathbb{T}$ and $n \in \mathbb{Z}_{+}$. Then

1. The nontangential boundary limits $w_{j}\left(t_{0}\right)$ exist for $j=0, \ldots, 2 n+1$ and $\left|w_{0}\left(t_{0}\right)\right|=1$.

2. The boundary Schwarz-Pick matrix $\mathbf{P}_{n}^{w}\left(t_{0}\right)$ exists and is equal to the matrix $\mathbb{P}_{n}^{w}\left(t_{0}\right)$ defined in (1.9). In particular, $\mathbb{P}_{n}^{w}\left(t_{0}\right)$ is Hermitian.

By Theorem 1.4, conditions (4.23) guarantee the existence of the the boundary Schwarz-Pick matrices $\mathbf{P}_{n}^{s}\left(t_{0}\right)$ and $\mathbf{P}_{n}^{f}\left(t_{0}\right)$. The rest is the same as in the proof of Theorem 4.2.

Note that in case when $w=\frac{s}{f}$ is the ratio of two Schur class functions, the condition

$$
\liminf _{z \rightarrow t_{0}} d_{w, n}(z)<\infty
$$

follows from (4.23) but does not imply (4.23) in general. Thus, the conclusions in Theorem 4.7 are obtained under apperantly too strong assumptions. Conditions (4.23) in Theorem 4.7 cannot be relaxed to (4.24) and we do not know what conditions in terms of $d_{w, n}(z)$ may replace (4.23).

\section{Infinite $t_{0}$-isometric sequences and related results}

Theorem 2.5 shows that any finite $t_{0}$-isometric sequence $\left\{w_{0}, \ldots, w_{n}\right\}$ arises as a sequence of the first $n+1$ Taylor coefficients of a rational unimodular function at $t_{0}$ (moreover, one can use rational unimodular functions with the only pole at the origin to get all finite $t_{0}$-isometric sequences. In this section we characterize infinite $t_{0}$-isometric sequences in similar terms.

Definition 5.1. We will say that a sequence $\left\{w_{j}\right\}_{j=0}^{\infty}$ of complex numbers is $t_{0}$ positive if $\left|w_{0}\right|=1$ and the matrix $\mathbb{P}\left(t_{0}, w_{0}, \ldots, w_{2 n+1}\right)$ defined as in (1.13) is positive semidefinite for all $n \geq 0$.

Remark 5.2. By Theorem 2.5, a sequence $t_{0}$-isometric if and only if $\left|w_{0}\right|=1$ and the matrix $\mathbb{P}\left(t_{0}, w_{0}, \ldots, w_{2 n+1}\right)$ is Hermitian for every $n \geq 0$. In partcular, any $t_{0}$-positive sequence is $t_{0}$-isometric. 
Definition 5.3. Let $t_{0} \in \mathbb{T}$. We will say that a Schur function $w$ belongs to the class $\mathcal{S}_{t_{0}}$ if

$$
\liminf _{z \rightarrow t_{0}} d_{w, n}(z)<\infty \text { for all } n \geq 0
$$

or equivalently, if

$$
\lim _{z \rightarrow t_{0}} d_{w, n}(z)<\infty \text { for all } n \geq 0
$$

where $d_{w, n}(z)$ is defined as in (1.5). We denote by $\mathcal{Q} \mathcal{S}_{t_{0}}$ the class of functions $w$ of the form $w=\frac{s}{f}$ where $s, f \in \mathcal{S}_{t_{0}}$.

Theorem 5.4. Let $t_{0} \in \mathbb{T}$ and let $w \in \mathcal{S}_{t_{0}}$. Then the nontangential boundary limits $w_{j}\left(t_{0}\right)$ exist for every $j \geq 0$ and the sequence $\left\{w_{j}\left(t_{0}\right)\right\}_{j=0}^{\infty}$ is $t_{0}$-positive. Furthermore, any $t_{0}$-positive sequence arises in this way.

Proof. The first assertion follows from Theorem 1.4 and Definition (5.1) of $t_{0^{-}}$ positivity. In regard to the second assertion recall a known interpolation result [10] (analogous to the classical Hamburger's solvability criteria for the power moment problem [8]) asserting that if a sequence $\left\{w_{j}\right\}_{j=0}^{\infty}$ is $t_{0}$-positive, then (an only then) there exists a Schur class function $w$ such that

$$
w_{j}\left(t_{0}\right):=\lim _{z \rightarrow t_{0}} \frac{w^{(j)}(z)}{j !}=w_{j} \text { for } j \geq 0 .
$$

For this function $w$ we have $\left|w_{0}\right|=1$ and $\mathbb{P}_{n}^{w}\left(t_{0}\right) \geq 0$ for all $n \geq 0$ and then it follows by Theorem 1.4 (implication $(4) \Rightarrow(2)$ that condition (1.16) holds for all $n \geq 0$, i.e., that $w \in \mathcal{S}_{t_{0}}$.

Theorem 5.5. Let $t_{0} \in \mathbb{T}$ and let $w \in \mathcal{Q S}_{t_{0}}$. Then the nontangential boundary limits $w_{j}\left(t_{0}\right)$ exist for every $j \geq 0$ and the sequence $\left\{w_{j}\left(t_{0}\right)\right\}_{j=0}^{\infty}$ is $t_{0}$-isometric. Furthermore, any $t_{0}$-isometric sequence arises in this way.

Proof. The first assertion follows from Theorem 4.7. To prove the second assertion, let us assume that $\left\{w_{j}\left(t_{0}\right)\right\}_{j=0}^{\infty}$ is a $t_{0}$-isometric sequence. Then by Remark 5.2, $\left|w_{0}\right|=1$ and $\mathbb{P}\left(t_{0}, w_{0}, \ldots, w_{2 n+1}\right)$ is Hermitian for every $n \geq 0$. Then we use the inductive construction from the proof of Lemma 2.4 to get a $t_{0}$-positive sequence $\left\{f_{j}\right\}_{j=0}^{\infty}$ such that the sequence $\left\{s_{j}\right\}_{j=0}^{\infty}$ defined by

$$
s_{j}=\sum_{\ell=0}^{j} f_{j-\ell} w_{\ell} \text { for } j=0,1, \ldots
$$

is $t_{0}$-positive. By Theorem 5.4, there exist functions $s(z)$ and $f(z)$ in $\mathcal{S}_{t_{0}}$ satisfying the conditions

$$
\frac{s^{(j)}\left(t_{0}\right)}{j !}=s_{j} \quad \text { and } \quad \frac{f^{(j)}\left(t_{0}\right)}{j !}=f_{j} \quad \text { for } j=0,1, \ldots
$$

Now it follows from (5.3) that the quotient $w(z)=\frac{s}{f}$ (which belongs to $\mathcal{Q S}_{t_{0}}$ ) satisfies the conditions (5.2). 
For general analytic functions we have the following result which is an infinite analogue of Theorem 1.5.

Theorem 5.6. Let $w$ be analytic in a neighborhood $\mathcal{U}_{t_{0}}$ of $t_{0} \in \mathbb{T}$. The following are equivalent:

1. The nontangential boundary limits $w_{j}\left(t_{0}\right)$ exist for every $j \in \mathbb{Z}_{+}$and the sequence $\left\{w_{j}\left(t_{0}\right)\right\}_{j=0}^{\infty}$ is $t_{0}$-isometric.

2. There exist a function $f \in \mathcal{Q S}_{t_{0}}$ such that

$$
w(z)=f(z)+o\left(\left(z-t_{0}\right)^{n}\right) \quad \text { for all } n \geq 0 \text { as } z \widehat{\rightarrow} t_{0} .
$$

3. $w$ admits the analytic continuation by symmetry $w(z)=\frac{1}{\overline{w(1 / \bar{z})}}$ across $t_{0}$ into an external nontangential neighborhood

$$
\widetilde{\Gamma}_{t_{0}, \alpha, \varepsilon}:=\left\{z \notin \mathbb{D}:\left|z-t_{0}\right|<\varepsilon,\left|\arg \left(z-t_{0}\right)\right|<\alpha\right\}
$$

of $t_{0}$ for every $\alpha \in\left(0, \frac{\pi}{2}\right)$ and $\varepsilon=\varepsilon(\alpha)>0$.

Proof. For the proof of $(1) \Leftrightarrow(3)$ it suffices to note that the nontangential asymptotic equality (1.19) holding for every $n \in \mathbb{Z}_{+}$from inside and outside of the unit disk, is equivalent to Statement 3 in Theorem 5.6. Equivalence (1) $\Leftrightarrow(2)$ follows from Theorem 5.5: since the limits $f_{j}\left(t_{0}\right)$ exist for $j \geq 0$, the relation (5.5) is equivalent to existence of the limits $w_{j}\left(t_{0}\right)=f_{j}\left(t_{0}\right)$ for $j \geq 0$.

\section{References}

[1] J. A. Ball, I. Gohberg and L. Rodman, Interpolation of rational matrix functions, Birkhäuser Verlag, Basel, 1990.

[2] V. Bolotnikov and H. Dym, On boundary interpolation for matrix Schur functions, to appear in Memoirs of AMS

[3] V. Bolotnikov and A. Kheifets, A higher order analogue of the Carathéodory-Julia theorem, J. Funct. Anal., to appear.

[4] V. Bolotnikov and A. Kheifets, On negative inertia of Pick matrices associated with generalized functions, Integral Equations Operator Theory, to appear.

[5] V. Bolotnikov and A. Kheifets, Carathéodory-Julia type theorems for operator valued Schur functions, Preprint.

[6] V. Bolotnikov and A. Kheifets, The Carathéodory-Julia theorem and related boundary interpolation problems, preprint.

[7] C. Carathéodory, Über die Winkelderivierten von beschränkten analytischen Funktionen, Sitz. Preuss. Akad. Phys.-Math., 4 (1929), 1-18.

[8] H. Hamburger, Über eine Erweiterung des Stieltjesschen Momentenproblems, Math. Ann. 82 (1921), no. 3-4, 168-187.

[9] G. Julia, Extension d'un lemma de Schwartz, Acta Math. 42 (1920), 349-355.

[10] A. Kheifets, Hamburger moment problem: Parseval equality and Arov-singularity, J. Funct. Anal. 141 (1996), no. 2, 374-420. 
[11] I. V. Kovalishina, A multiple boundary interpolation problem for contractive matrixvalued functions in the unit circle, Teoriya Funktsii, Funktsianal'nyi Analiz i Ikh Prilozheniya, 51 (1989), 38-55. English transl. in: Journal of Soviet Mathematics, 52(6) (1990), 3467-3481.

[12] M. G. Krĕn and H. Langer, Über die verallgemeinerten Resolventen und die charakteristische Funktion eines isometrischen Operators im Raume $\Pi_{\kappa}$, Colloq. Math. Soc. János Bolyai 5 (1972), 353-399.

[13] M. G. Krel̆n and H. Langer, Über einige Fortsetzungsprobleme, die eng mit der Theorie hermitescher Operatoren im Raume $\Pi_{\kappa}$ zusammenhängen. I. Einige Funktionenklassen und ihre Darstellungen Math. Nachr. 77 (1977), 187-236.

[14] D. Sarason, Angular derivatives via Hilbert space, Complex Variables Theory Appl., 10(1) (1988), 1-10.

[15] D. Sarason, Sub-Hardy Hilbert Spaces in the Unit Disk, Wiley, New York, 1994.

[16] J. H. Shapiro, Composition operators and classical function theory, Springer-Verlag, New York, 1993.

Vladimir Bolotnikov and Alexander Kheifets

Department of Mathematics, The College of William and Mary, Williamsburg, VA 231878795, USA

e-mail: vladi@math.wm.edu

Department of Mathematics, University of Massachusetts Lowell, Lowell, MA, 01854, USA

e-mail: Alexander_Kheifets@uml.edu 